\title{
Genome-wide identification and expression profiling of Alba gene family members in response to abiotic stress in tomato (Solanum lycopersicum L.)
}

\author{
Antt Htet Wai ${ }^{1,2}$, Lae-Hyeon Cho ${ }^{3}$, Xin Peng ${ }^{4}$, Muhammad Waseem5 ${ }^{5}$ Do-jin Lee ${ }^{1}$, Je-Min Lee ${ }^{6}$, \\ Chang-Kil Kim ${ }^{6}$ and Mi-Young Chung ${ }^{1 *}$
}

\begin{abstract}
Background: Alba (Acetylation lowers binding affinity) proteins are an ancient family of nucleic acid-binding proteins that function in gene regulation, RNA metabolism, mRNA translatability, developmental processes, and stress adaptation. However, comprehensive bioinformatics analysis on the Alba gene family of Solanum lycopersicum has not been reported previously.

Results: In the present study, we undertook the first comprehensive genome-wide characterization of the Alba gene family in tomato (Solanum lycopersicum L.). We identified eight tomato Alba genes, which were classified into two groups: genes containing a single Alba domain and genes with a generic Alba domain and RGG/RG repeat motifs. Cis-regulatory elements and target sites for miRNAs, which function in plant development and stress responses, were prevalent in SIAlba genes. To explore the structure-function relationships of tomato Alba proteins, we predicted their 3D structures, highlighting their likely interactions with several putative ligands. Confocal microscopy revealed that SIAlba-GFP fusion proteins were localized to the nucleus and cytoplasm, consistent with putative roles in various signalling cascades. Expression profiling revealed the differential expression patterns of most SIAlba genes across diverse organs. SIAlba1 and SIAlba2 were predominantly expressed in flowers, whereas SIAlba5 expression peaked in $1 \mathrm{~cm}$-diameter fruits. The SIAlba genes were differentially expressed (up- or downregulated) in response to different abiotic stresses. All but one of these genes were induced by abscisic acid treatment, pointing to their possible regulatory roles in stress tolerance via an abscisic acid-dependent pathway. Furthermore, co-expression of SIAlba genes with multiple genes related to several metabolic pathways spotlighted their crucial roles in various biological processes and signalling.
\end{abstract}

Conclusions: Our characterization of SIAlba genes should facilitate the discovery of additional genes associated with organ and fruit development as well as abiotic stress adaptation in tomato.

Keywords: Alba genes, Solanum lycopersicum, Abiotic stress, Expression profiling, Subcellular localization, 3D structure, Gene co-expression network

*Correspondence: queen@sunchon.ac.kr

${ }^{1}$ Department of Agricultural Education, Sunchon National University, 413

Jungangno, Suncheon, Jeonnam 540-950, Republic of Korea

Full list of author information is available at the end of the article

\section{Background}

Alba (Acetylation lowers binding affinity) superfamily proteins belong to an ancient group of nucleic acidbinding proteins that originated prior to the divergence 
of archaea and eukaryotes and are widely distributed in nearly all kingdoms of life $[1,2]$. These small, basic proteins interact with DNA and RNA as homodimers or heterodimers [3-6] and contain a conserved nucleic acid-binding Alba domain with an IF3-C fold [1]. Alba proteins also show the distinct characteristic of being regulated via acetylation by PAT (protein acetyl transferase) and deacetylation by Sir2 (an $\mathrm{NAD}^{+}$-dependent histone deacetylase, HDAC), indicating that they function as transcriptional regulators similar to histones [7-11]. Although Alba proteins were initially classified as chromosomal proteins and were considered to function in the maintenance of chromatin architecture and transcriptional repression, many studies have documented their functional diversity, including their roles in genome packaging and organization, transcriptional and translational regulation, RNA metabolism, and development and differentiation processes [2, 12-14].

Alba proteins exist in two forms: relatively small proteins with a single generic Alba domain; and larger proteins with an Alba domain plus RGG/RG repeat motifs or additional domain/s [2, 15]. All archaeal Alba homologs, which belong to the Archaea Alba protein family, are relatively small proteins with only a single Alba domain. Eukaryotic Alba proteins diverged into two paralogous lineages, the Rpp20-like family (with single Alba domains) and the Rpp25-like family (mostly larger proteins with RGG/RG repeat motifs or additional domains) [1]. The RGG/RG repeat motifs are thought to have roles in DNA damage signalling, snRNP biogenesis, the regulation of apoptosis, transcription, pre-mRNA splicing, and translation, many of which are at least partly controlled by the arginine methylation of RGG/ RG repeats [16].

Alba genes encoding proteins with RGG/RG repeat motifs have been identified in many species $[15,17,18]$. Various types of domains with diverse biological functions have also been identified in combination with the Alba domain in numerous Alba family proteins in many domains of life, such as the F-box domain in the fungus Taphrina; the CLIP1 zinc finger domain in the nematode Pristionchus; the ATP synthase subunit $\mathrm{H}$ domain in the protozoan parasite Theileria; the sugar transporter domain in the Florida lancelet; the DEAD/DEAH box helicase domain in plants; and so forth [2]. The addition of RGG/RG repeat motifs and new domains to genes with a single Alba domain appears to have contributed to speciation and the functional diversification of Alba family proteins to meet the demands of increasing cellular variety and complexity.

Diverse abiotic stresses, including drought, salinity, heat, and cold stress, can hamper plant growth and development, resulting in significantly reduced crop productivity [19]. Various environmental stresses trigger changes in Alba behaviour. For example, LiAlba1 and LiAlba3, from the protozoan parasite Leishmania infantum, repress translation by interacting with RNA-binding proteins and ribosomal subunits. These proteins also function as translation factors and translocate from the cytoplasm into the nucleolus and flagellum in response to heat stress [20]. Similarly, all four Alba proteins of Trypanosoma brucei (TbAlba1, TbAlba2, TbAlba3, and TbAlba4) are RNA-binding proteins that localize to the cytoplasm as parts of stress granules (SG) upon exposure to nutrient stress [21]. The stress-induced differential expression has also been observed for Alba genes from rice treated with different abiotic stresses and phytohormones [22]. Mild heat stress $\left(37^{\circ} \mathrm{C}\right)$ altered the expression of most Arabidopsis Alba genes in inflorescences [15]. In cotton (Gossypium hirsutum), GhALBA4 and GhALBA5 were significantly induced by water deficit and salinity treatment, and plants in which the expression of these genes was repressed by virus-induced gene silencing (VIGS) were highly sensitive to dehydration as well as salt stress, pointing to their putative roles in abiotic stress tolerance [23].

Genome-wide identification of Alba genes has been performed in several plant species, but no comprehensive study of the evolutionary relationships or characteristics of the Alba gene family in solanaceous crops has been reported. Tomato (Solanum lycopersicum L.) is an economically important fruit whose yields are severely affected by unfavorable environmental conditions [19]. The molecular mechanisms regulating fruit development and ripening have been extensively studied in this model fruit crop with the aim of increasing fruit yield under diverse environmental stresses. Hence, in the current study, we performed a comprehensive genome-wide analysis, gene co-expression network analysis and expression profiling of SlAlba genes in response to various abiotic stresses. Our findings provide a basis for further functional characterization of Alba genes involved in the development and stress tolerance of tomato.

\section{Results \\ Identification and sequence analysis of Alba family proteins in tomato}

We identified eight non-redundant putative Alba genes in tomato, which were named SlAlba1 to SlAlba8 in accordance with their positions on the chromosomes. The open reading frames of the SlAlba genes showed considerable variation in length, ranging from 384 bp to $1188 \mathrm{bp}$ for SlAlba5 and SlAlba7, respectively, with a mean of $701 \mathrm{bp}$. The predicted sizes of the eight SlAlba proteins varied from 127 (SlAlba5) to 395 (SlAlba7) 
amino acids (aa), with a mean of 236 aa. The computed molecular weights of these proteins ranged from 14.24 to 43.29 with iso-electric points (pI) of 5.61 to 10.22 . Based on their predicted pI values, the SlAlbas include both acidic and basic proteins. The grand average of hydropathicity (GRAVY) values of these proteins ranged from -1.126 to -0.312 , indicating that they all are hydrophilic proteins (Table 1 ).

\section{Phylogenetic and domain analysis of tomato Alba proteins} Phylogenetic analysis clearly placed Alba family members from multiple diverse species into three major families: one archaea-specific family and two eukaryote-specific families, the RPP20-like and RPP25-like families (Fig. 1). The archaea-specific family solely comprises archaeal Alba proteins, whereas the eukaryotic homologs of Alba are organized into two distinct families. We constructed an evolutionary tree, which revealed a major cluster containing proteins from dicots and monocots together with lower plants. The tomato Alba proteins were distributed in both eukaryote-specific families, with the largest number of SlAlbas being RPP25-like family proteins with RGG/RG repeat boxes. The remaining SlAlbas lacked RGG/RG motifs and were grouped in the RPP20-like family; no SlAlba protein was found in the archaea-specific family. Phylogenetic tree analysis indicated that the Alba members from tomato were selectively paired with those from the closely related solanaceous crop potato in both eukaryotic families.

Interestingly, the RPP20-like Alba family members from the basal angiosperm Amborella showed a close evolutionary relationship with the corresponding proteins from the single-celled green alga Chlamydomonas reinhardtii and dicotyledonous grapevine, whereas the RPP25-like proteins of Amborella were grouped in the same clades as those of monocotyledonous crop species. Alba homologs from the moss Physcomitrella
(PpAlb2, PpAlb3, and PpAlba4) were clustered together and showed closer relationships with those of solanaceous crops in the RPP25-like family, whereas the RPP20-like protein (PpAlb1) was clustered together with those of cereal crops. In the archaeal Alba protein group, SsoAlba1 and SsoAlba2 from Sulfolobus solfataricus were paired with their related orthologs SshAlba1 and SshAlba2, respectively, from Sulfolobus shibitae. However, Archaeoglobus fulgidus (AfAlba1) and Aeropyrum pernix (ApAlba1) were grouped in the same clades with their paralogs derived from lineage-specific evolution (AfAlba2 and ApAlba2). These results point to the diversification of Alba proteins across various kingdoms of life as well as the sequence conservation in related groupings during the evolutionary process.

Of the eight Alba proteins identified in tomato, five (SlAlba2, SlAlba3, SlAlba6, SlAlba7, and SlAlba8) displayed a long-form structure comprising an $\mathrm{N}$-terminal Alba domain followed by a C-terminal region with several arginine-glycine (RGG) repeats, which function as RNA recognition motifs to interact particularly with guanine (G)-quadruplexes in RNA [24, 25]. However, these motifs were absent from the three remaining, shortform SlAlba proteins (SlAlba1, SlAlba4, and SlAlba5) (Fig. 2). The clustering of the long-form SlAlba proteins and short-form SlAlba proteins in different eukaryotic families highlights the importance of RGG repeats in the structural and functional diversification of tomato Alba proteins during the course of evolution.

All Alba proteins from tomato and potato belonging to the RPP20-like family share a conserved NRIQVShexapeptide motif at the start of their Alba domains, whereas most of their homologs from the RPP25-like family contain a distinct conserved NEIRIT-hexapeptide motif (Fig. 3). These findings indicate that these motifs were conserved in tomato and potato and are

Table 1 Characteristics of the Alba genes and corresponding proteins in tomato genome

\begin{tabular}{|c|c|c|c|c|c|c|c|c|c|c|}
\hline \multirow[t]{2}{*}{ Gene Name } & \multirow[t]{2}{*}{ Locus name } & \multirow[t]{2}{*}{ ORF (bp) } & \multirow{2}{*}{$\begin{array}{l}\text { Chromosome } \\
\text { No. }\end{array}$} & \multicolumn{5}{|l|}{ Protein } & \multirow{2}{*}{$\begin{array}{l}\text { Subcellular } \\
\text { Localization }\end{array}$} & \multirow{2}{*}{$\begin{array}{l}\text { No. of } \\
\text { Introns }\end{array}$} \\
\hline & & & & Length (aa) & $\begin{array}{l}\text { Alba Domain } \\
\text { Start-End (aa) }\end{array}$ & MW (kDa) & $\mathrm{PI}$ & GRAVY & & \\
\hline SIAlbal & Solyc01g097050 & 498 & 1 & 193 & $67-130$ & 21.20 & 9.7 & -0.312 & Chloroplast & 3 \\
\hline SIAlba2 & Solyc04g071690 & 702 & 4 & 233 & $19-85$ & 25.17 & 9.86 & -0.709 & Cytoplasm & 5 \\
\hline SIAlba3 & Solyc04g081880 & 765 & 4 & 254 & $19-84$ & 28.01 & 10.22 & -0.965 & Cytoplasm & 6 \\
\hline SIAlba4 & Solyc06g065980 & 387 & 6 & 128 & $20-84$ & 14.41 & 5.61 & -0.548 & Cytoplasm & 4 \\
\hline SIAlba5 & Solyc06g068050 & 384 & 6 & 127 & $20-85$ & 14.24 & 6.32 & -0.373 & Chloroplast & 4 \\
\hline SIAlba6 & Solyc06g083540 & 789 & 6 & 262 & $19-82$ & 29.23 & 9.95 & -1.126 & Cytoplasm & 6 \\
\hline SIAlba7 & Solyc09g061710 & 1188 & 9 & 395 & $19-84$ & 43.29 & 9.28 & -0.826 & Cytoplasm & 8 \\
\hline SIAlba8 & Solyc09g091590 & 897 & 9 & 303 & $24-89$ & 32.90 & 9.68 & -1.032 & Nucleus & 7 \\
\hline
\end{tabular}

ORF Open reading frame, $b p$ Base pair, aa Amino acid, $k D a$ Kilo Dalton, $p /$ Isoelectric point, MW Molecular weight, GRAVY Grand average of hydropathicity 


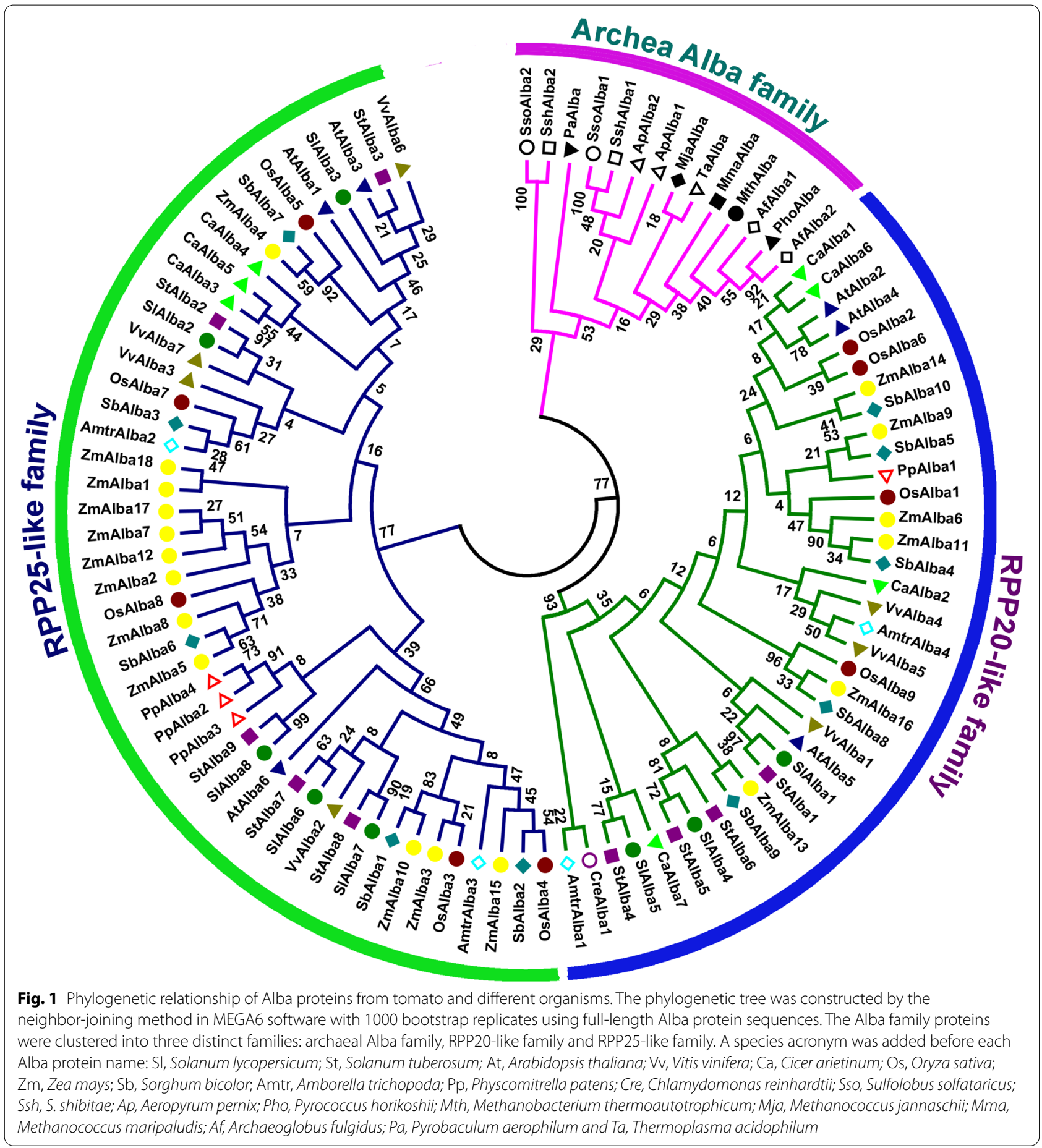

related to the structural and functional similarity of Alba family members from solanaceous crops. Multiple sequence alignment of SlAlba proteins with a well-characterized Alba homolog from the human malaria parasite Plasmodium falciparum highlighted the evolutionary conservation of RPP-20-like family
Alba proteins from a unicellular parasite and a flowering plant, particularly the presence of similar amino acid residues in the hexapeptide motif at the start of the Alba domain and in the TKKP-tetrapeptide motif of the first loop (L1), which are related to the DNA binding affinity of the Alba domain (Fig. 3). 


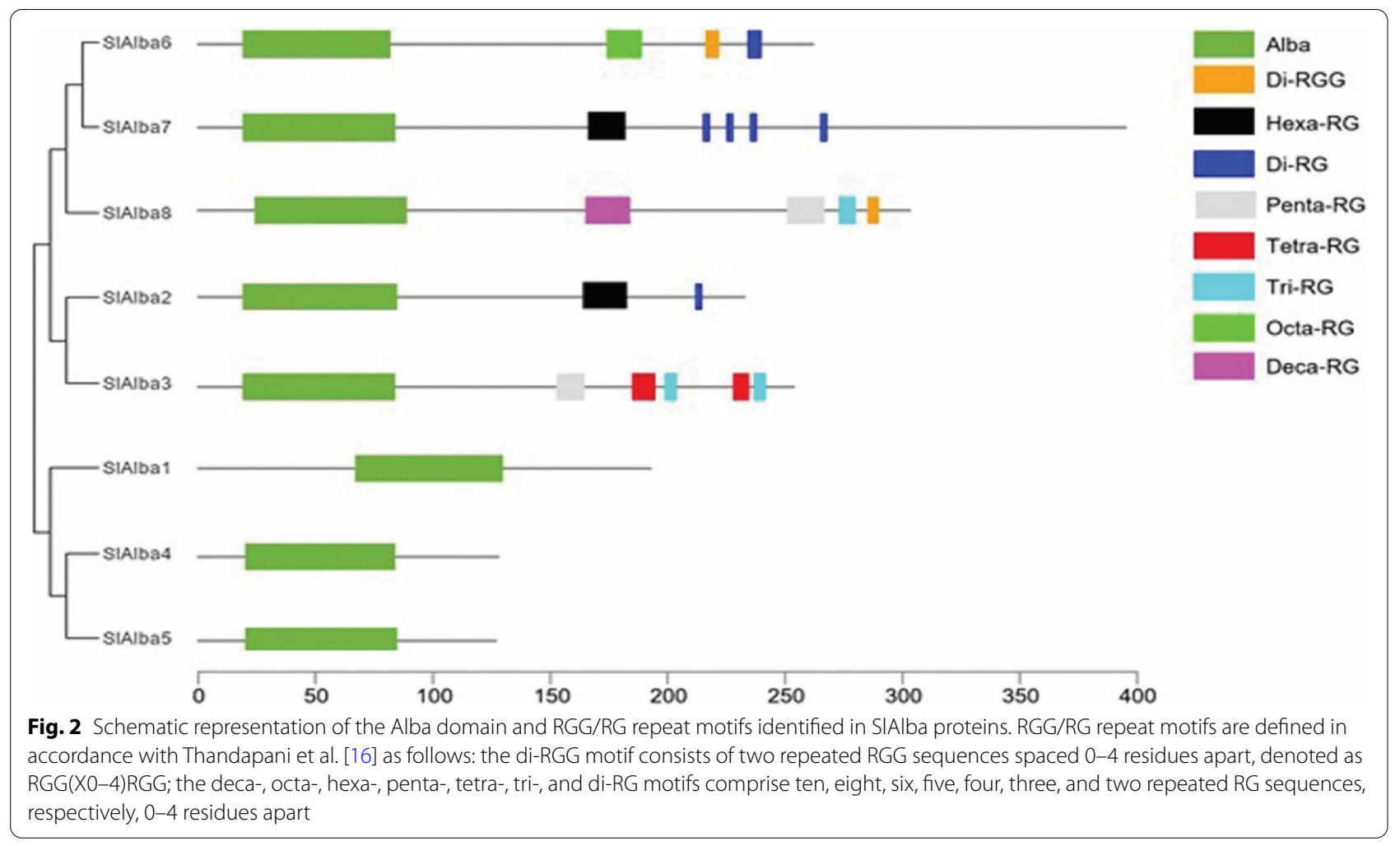

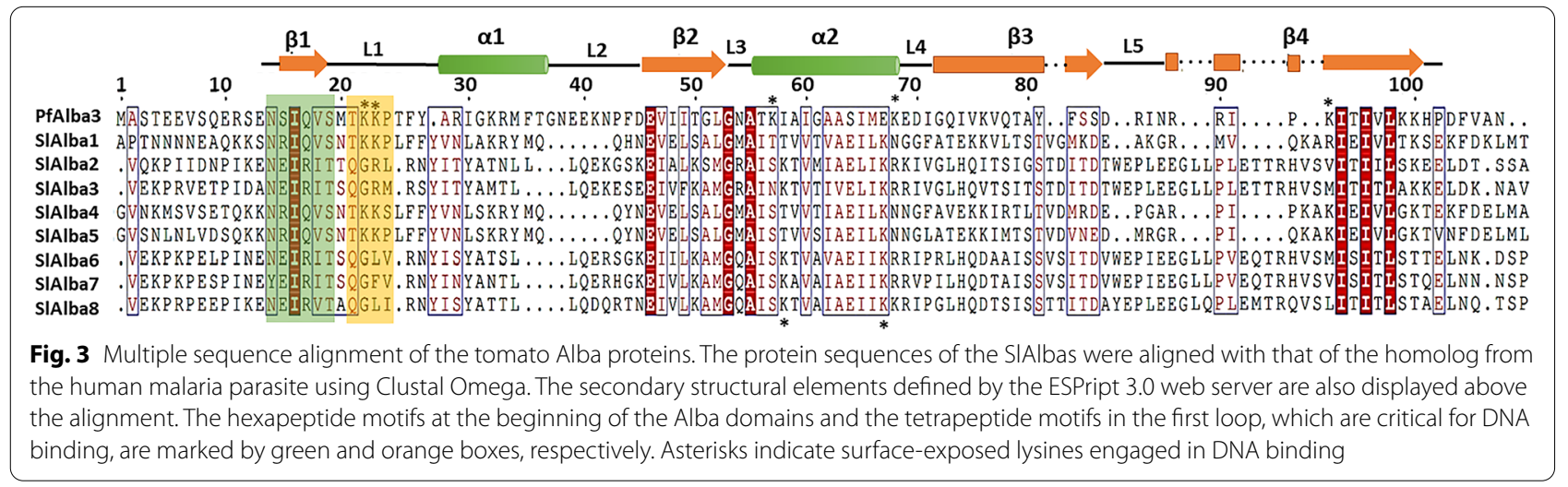

\section{Analysis of the exon-intron structures and conserved motifs of SIAlba genes}

Analysis of the exon-intron structures of the SlAlba genes showed that the numbers of exons (4 or 5 ) in tomato Alba genes in the RPP20-like family were almost identical. By contrast, members of the RPP25-like family contained 6 to 9 exons, with an average of 7 (Fig. S1).

SlAlba7 had the most exons (9), while SlAlba1 had the fewest (4). However, the genes in the same phylogenetic group shared similar exon-intron compositions in terms of intron number and exon size.
In-depth motif analysis revealed that Alba proteins from the RPP20-like family had fewer motifs (3 or 4) than those from the RPP25-like family (7 to 14 with a mean of 9) (Figs. S2 and S7). Motifs 1 and 2 in the Alba domain were conserved in all Alba proteins, while motifs 7, 8, and 13, which contain the RGG/RG repeat motif, were prevalent only in large Alba proteins belonging to the RPP25like family. All RPP20-like family members shared similar motif structures and compositions, containing motif 3 , which is characteristic of the members of the RPP20-like family, in addition to motifs 1 and 2. These findings 
highlight the importance of these motifs in the evolutionary conservation of Alba proteins from this family in both monocot and dicot plants. Motifs 1, 2, 4, 5, and 6 were highly conserved in all members of the RPP25-like family, pointing to their role in the structural similarity among Alba members belonging to this group in dicots and monocots. Several conserved motifs were unique to certain RPP25-like family members, such as motif 9 for SlAlba7, motif 11 for SlAlba1 and OSAlba7, motif 12 for SlAlba6 and SlAlba7, motif 14 for SlAlba8 and OsAlba7, and motif 15 for OsAlba5 and OsAlba7. Interestingly, motif 10 was absent from Arabidopsis but present in many rice and tomato family members. Among the conserved motifs identified in Alba proteins, motif7, motif8, and motif13, all of which are composed of RG rich repeats, play a role in the interaction with nucleic acids and proteins [16].

\section{Chromosomal distribution, gene duplication, and microsynteny analysis}

The eight tomato Alba genes were distributed on four of the 12 tomato chromosomes $(1,4,6$, and 9), with all genes residing close to the distal ends of the chromosomes. The SlAlba genes appeared to be distributed unevenly among the chromosomes, with a single gene on chromosome 1, two genes each on chromosomes 4 and 9, and three genes on chromosome 6 (Fig. S3).

Of the eight SlAlba genes, only SlAlba6 and SlA$l b a 7$ were predicted to be a segmentally duplicated gene pair; these genes are located on chromosomes 6 and 9, respectively (Fig. S4). No tandemly duplicated genes were predicted in the SlAlba gene family since no genes resided within a $100-\mathrm{kb}$ distance on the same chromosome. SlAlba proteins from the same phylogenetic clades exhibited higher sequence identity compared to those from different clades (Table S4). The Ka/ Ks ratio of the duplicated gene pair (SlAlba6/SlAlba7) was $>1$, indicating that these genes had undergone positive selection during the process of evolution. The predicted divergence time of the paralogous gene pair (SlAlba6/SlAlba7) indicates that the gene duplication event took place 64.09 million years ago (MYA) (Table 2). We constructed a comparative microsynteny map to analyze orthologous Alba gene pairs from tomato, rice, and Arabidopsis in order to investigate the evolutionary history and relationships across their genomes. This analysis predicted three orthologous gene pairs between tomato and Arabidopsis, but only one orthologous gene pair between tomato and rice, as well as between Arabidopsis and rice (Fig. 4).

\section{Analysis of putative stress- and hormone-responsive cis-elements and miRNA target sites in SIAlba genes}

To assess the transcriptional regulation of SlAlba genes under abiotic stress conditions, we analyzed the 1500bp sequences in their promoter regions. We identified various numbers of phytohormone- and stress-responsive cis-regulatory elements in the promoters of SlA$l b a$ genes, including the following: TC-rich repeats (implicated in defence and stress responses); droughtresponsive MYB-binding site (MBS); cold- and hypersalinity-responsive low temperature-responsive elements (LTRs); the CGTCA-motif (associated with the jasmonic acid [JA] response); ABRE elements (related to the ABA response); gibberellic acid (GA) response-related TATC-elements and GARG-motifs; TCA-elements (involved in the SA response); WUN motifs (with roles in wounding responses); and TGA elements and AuxRR-core (involved in the auxin response; Fig. S5 and Table S5). We also identified the target sites for several different types of miRNAs involved in various biological processes in the SlAlba genes (Table S6), many of which are related to development and stress responses, such as sly-miR94773p, sly-miR5303, sly-miR9474-5p, sly-miR9471a-3p, sly-miR9473-3p, miR156, miR167, miR169, miR171, miR172, miR393, miR395, miR396, miR397, miR399, and miR408, and miR827 [26-29].

\section{Prediction of the three-dimensional structures and functions of SIAlba proteins}

We predicted the 3D structures of SlAlba proteins using the optimal templates listed in Table S7 and other relevant information, such as the percentage of sequence identity, coverage, and Z-scores within the reliable range. An analysis of the resulting models with Discovery Studio v.21.1 revealed the putative binding sites, varied numbers of $\alpha$-helices, $\beta$-strands, and coils in the SlAlba proteins, as shown in Table S8 and Fig. 5. TM-scores of $<0.17$ and $>0.5$ indicate models with random similarity and a high level of homology, respectively. The TM-scores and RMSD values of the models fell within the reliable range,

Table 2 Estimated Ka/Ks ratio of the duplicated SIAlba gene pair with its divergence time in tomato

\begin{tabular}{lllllll}
\hline Duplicated gene pair & Ka & Ks & Ka/Ks & Duplication type & Types of selection & Time (MYA) \\
\hline SIAlba6 vs. SIAlba7 & 2.188636 & 1.922748 & 1.138286 & Segmental & Positive selection \\
\hline
\end{tabular}

Ks The number of synonymous substitutions per synonymous site, Ka The number of non-synonymous substitutions per nonsynonymous site, MYA Million years ago 


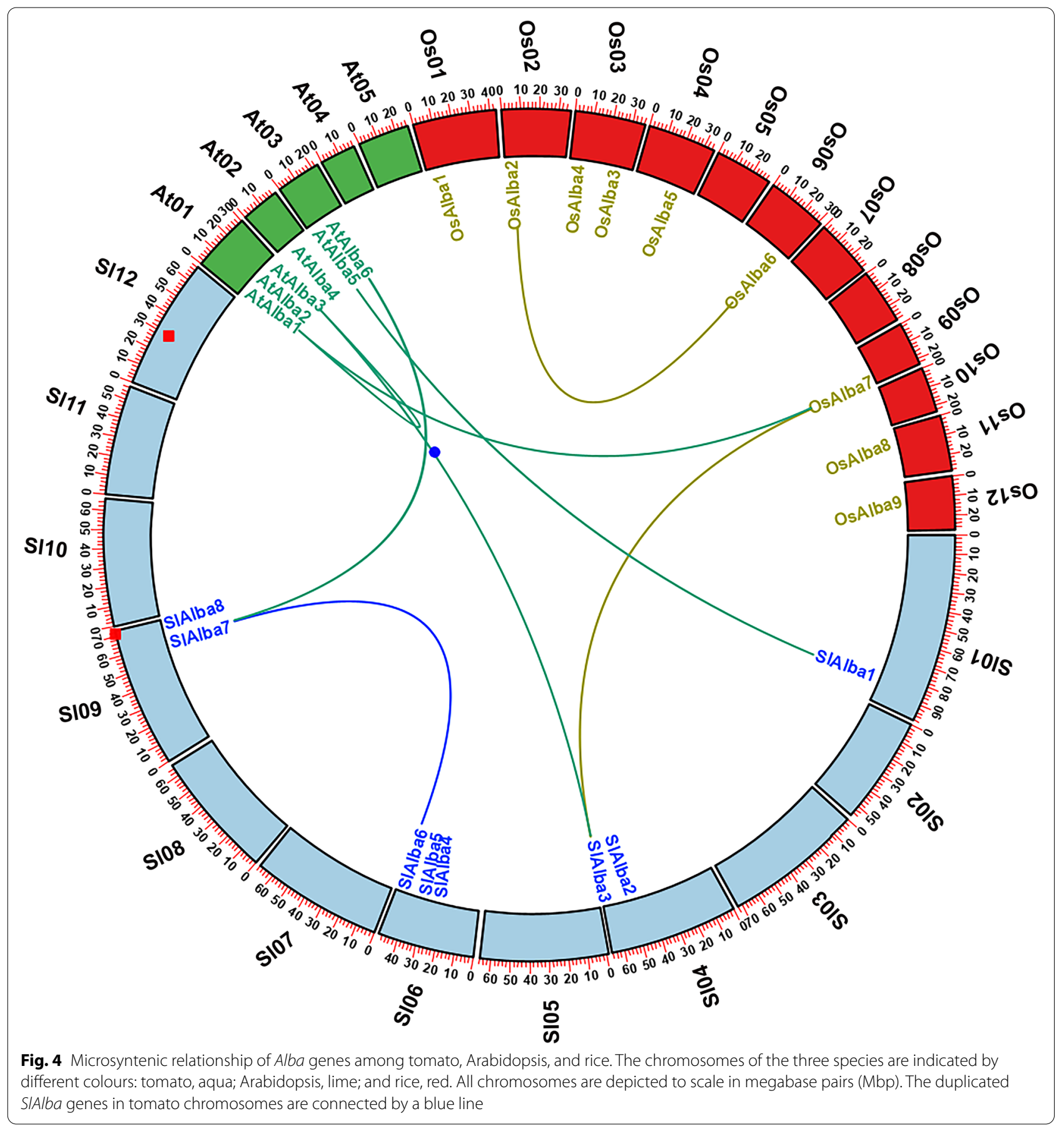

implying that the models were accurate (Table S9). The $\mathrm{C}$-scores of the predicted models ranged from -4.30 (SlAlba8) to -0.70 (SlAlba4), and other parameters, such as the number of decoys and cluster density, demonstrated that the results were within a credible range (Table S10).

We investigated the binding residues for the SlAlba proteins based on an alignment between the template and the newly generated models. Five of the eight SlAlba proteins (SlAlba1, SlAlba2, SlAlba4, SlAlba5, and SlAlba8) were predicted to bind to both DNA and RNA in addition to other ligands, such as peptides, arginine, and manganese. SlAlba3 and SlAlba7 displayed no affinity for nucleic acids, but they did have affinity for other ligands, such as $\beta$-carotene and magnesium, whereas SlAlba6 exhibited a specific affinity for RNA (Fig. S6). 
A

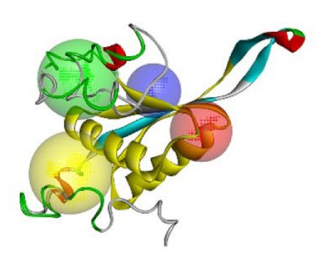

D

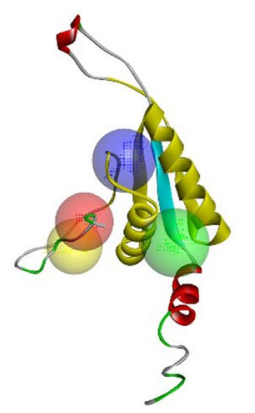

B

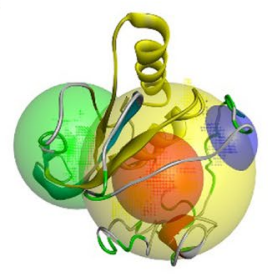

$\mathbf{E}$

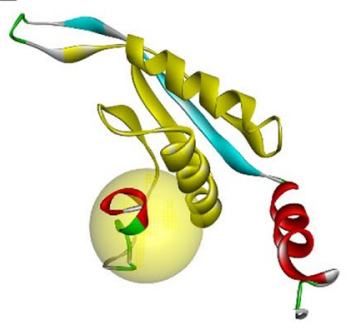

C

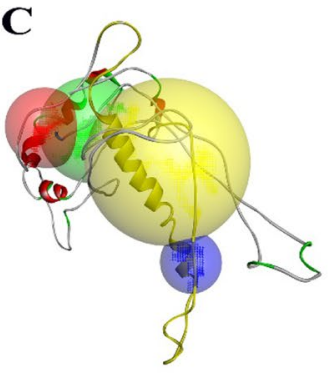

F

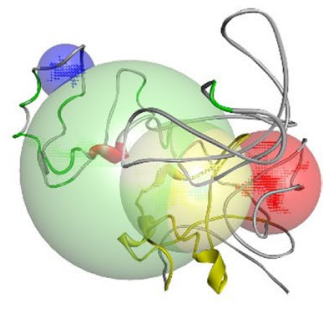

G

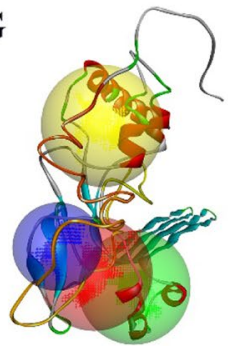

H

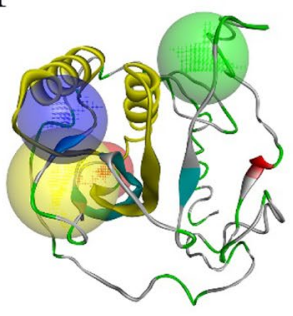

Fig. 5 The final 3-dimensional model structures of SIAlba proteins generated by Discovery Studio v.21.1.The secondary structural elements: a-helices (red), $\beta$-sheets (cyan), coils (green), and loops (gray) as well as the top four predicted binding sites: site 1 (yellow sphere), site 2 (green sphere), site 3 (red sphere), and site 4 (blue sphere) are depicted for the generated 3D model structures of A SIAlba1; B SIAlba2; C SIAlba3; D SIAlba4; E SIAlba5; F SIAlba6; G SIAlba7; and $\mathbf{H}$ SIAlba8. Alba domain regions in the predicted models are marked with yellow colour

Gene ontology (GO) analysis suggested that the tomato Alba family members play different roles in diverse biological processes, including chromosome condensation, cellular response to stress, and carbohydrate metabolic process, a putative role in cytolysis and protein complex oligomerization, and rRNA methylation (Table S12).

\section{Expression analysis of SIAlba genes in different organs}

Expression profiling revealed that most SlAlba genes showed differential expression patterns across the various organs investigated. Four out of the eight Alba genes were predominantly expressed in stem tissues, while two other Alba genes were preferentially expressed in flowers and the remaining two genes showed maximum expression in $1 \mathrm{~cm}$ fruits and leaves, respectively.

SlAlba1 was highly expressed in flowers at various stages of development, such as floral buds (550-fold relative to roots), full blooming flowers (800-fold relative to roots), and senescent flowers (250-fold relative to roots), whereas it displayed minimal expression in roots and $1 \mathrm{~cm}$ fruits and no expression in other organs. By contrast, homologs of SlAlba1 in the same phylogenetic group, SlAlba4 and SlAlba5, were broadly expressed across all organs examined. SlAlba4 showed the highest expression in stems (3-fold vs. the control [leaves]), but its transcript levels were significantly lower (2-fold to 4-fold) in fruits at all stages of development except for $1 \mathrm{~cm}$ fruits compared to the control. SlAlba5 was 3- to 8.5-fold more highly expressed in fruits at all six developmental stages except immature fruits compared to the control. The expression of SlAlba5 peaked (8.5-fold vs. the control) in $1 \mathrm{~cm}$ fruits, followed by stems ( $>5$-fold relative to the control).

SlAlba2, belonging to the RPP25-like family, is another flower-specific gene. This gene showed 

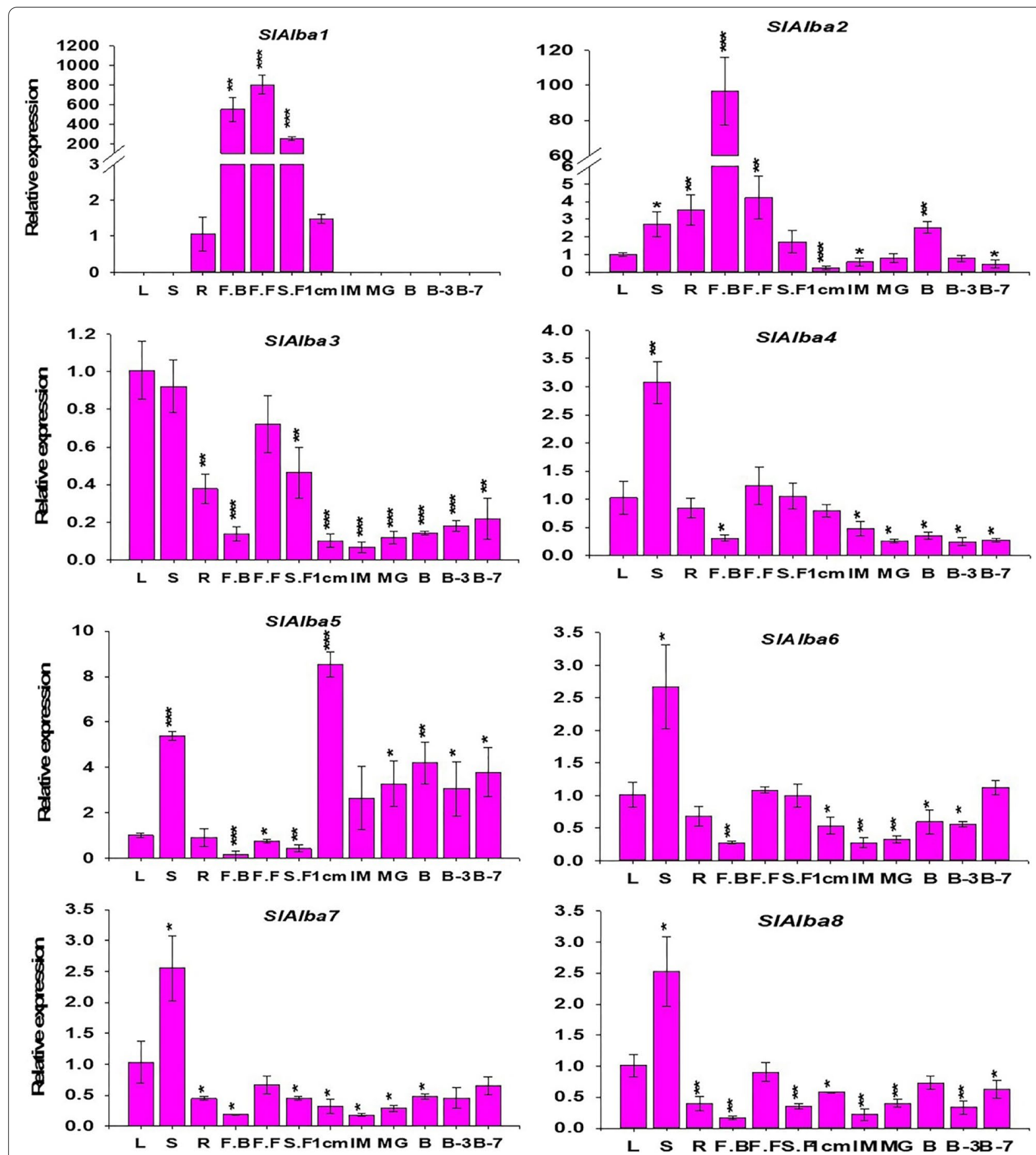

Fig. 6 Quantitative reverse-transcription PCR (RT-qPCR) analysis of SIAlba genes in 12 organs: leaves, roots, stems, flower buds (FB), full blooming flowers (FF), senescent flowers (SF), $1 \mathrm{~cm}$ fruits, immature fruits (IM), mature green fruits (MG), breaker fruits (B), fruits at 3 days after the breaker stage (B3), and fruits at 7 days after the breaker stage (B7). Error bars represent the standard deviations of the means of three independent replicates. *, ${ }^{*}$, and *** indicate the significant differences between the control samples (roots for SIAlbal and leaves for the remaining SIAlba genes) and the samples collected from the other organs, as determined by Student's t-test, at $p$-values $\leq 0.05, \leq 0.01$, and $\leq 0.001$, respectively 
maximum expression in flower buds (>96-fold higher than the control), followed by full blooming flowers (>4-fold compared with the control). The expression of SlAlba2 was 2.5-fold higher in breaker fruits than the control but significantly downregulated in $1 \mathrm{~cm}$ fruits, immature fruits, and B7 fruits during fruit development ( -1.7 to 4 -fold vs. the control). Intriguingly, SlAlba3 was the only gene whose transcripts were most abundant in leaves, followed by stems, and its expression was significantly reduced (by 4.5 - to 14.8 -fold relative to the control) throughout all stages of fruit development. SlAlba6, SlAlba7, and SlAlba8, homologous genes belonging to the same phylogenetic clade, exhibited a 2 -fold upregulation in stem tissues. Compared to the control, their transcript levels were significantly reduced in fruits during most stages of development (Fig. 6).
Expression analysis of tomato Alba family genes under different abiotic stresses and phytohormone treatments

SlAlba1 transcripts were undetectable in all leaf samples from control, abiotic stress-, or ABA-treated plants, whereas many of the other Alba genes displayed diverse expression patterns over time following exposure to these treatments (Fig. 7a-e).

Heat stress triggered significant changes in the transcript profiles of the SlAlba genes at various time points (Fig. 7a). Compared to the control (0h), SlAlba4 and SlAlba5 expression significantly increased (1.6-to 2 -fold) at $24 \mathrm{~h}$ after heat treatment. SlAlba5 expression was repressed (1.7-to 2-fold) during the early phases of heat stress but gradually recovered after $9 \mathrm{~h}$ and was upregulated (2-fold) at $24 \mathrm{~h}$ after heat stress. By contrast, the majority of Alba genes (SlAlba2, SlAlba3, SlAlba6,
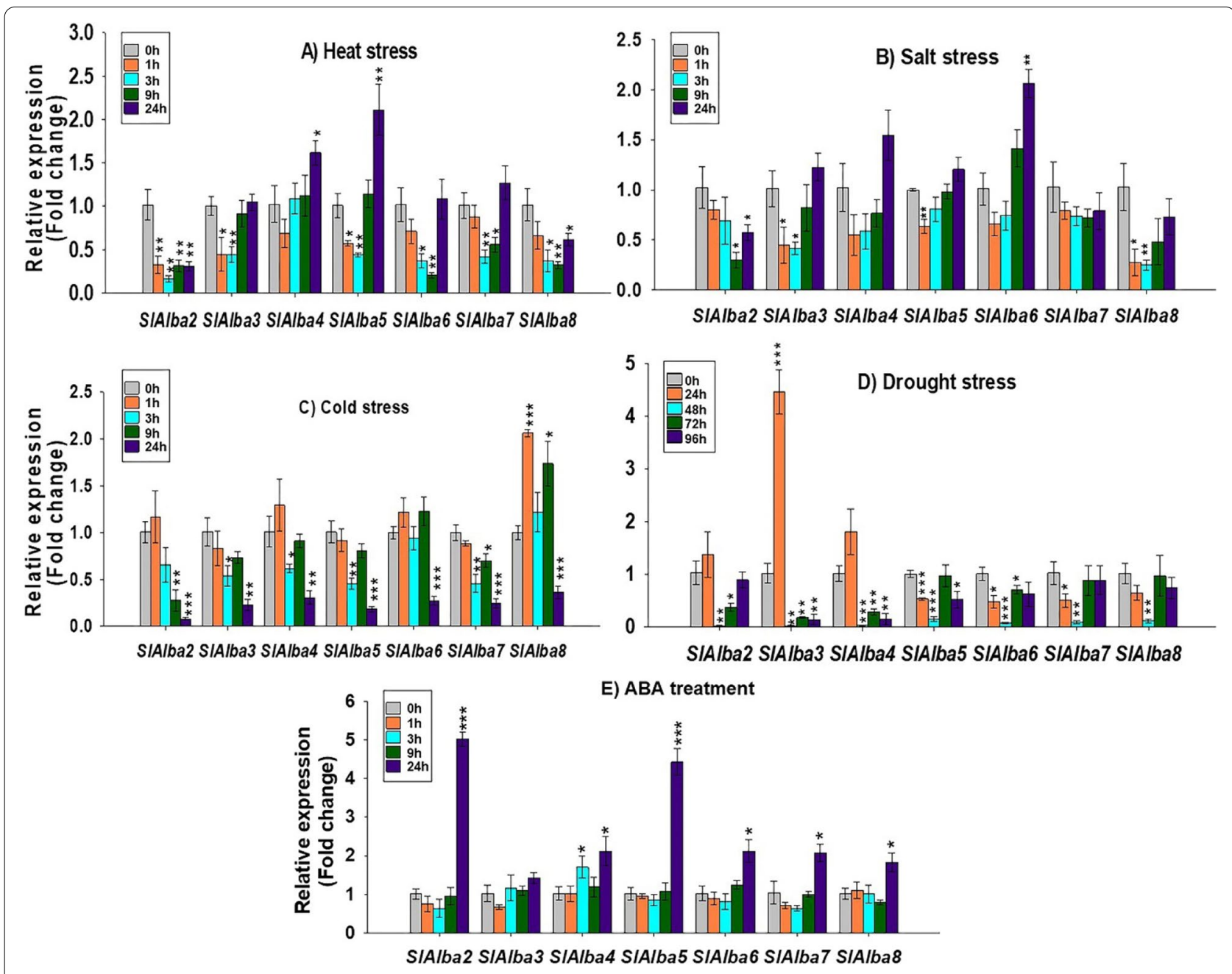

Fig. 7 Expression analysis of SIAlba genes by RT-qPCR under different abiotic stresses: a heat, b salt ( $\mathrm{NaCl}$ ), $\mathbf{c}$ cold, $\mathbf{d}$ drought, and e ABA treatment. Error bars denote standard deviations of the means of three independent replicates. The asterisks indicate significant differences, as determined by Student's t-test ( ${ }^{*} p$-value $\leq 0.05,{ }^{* *} p$-value $\leq 0.01$, and ${ }^{* * *} p$-value $\leq 0.001$ ) 
SlAlba7, and SlAlba8) showed similar responses to heat treatment, with decreased expression levels (1.6-fold to 6-fold vs. the control) following heat exposure.

Most of the Alba genes were responsive to salt treatment but SlAlba4 and SlAlba7 showed no significant change in expression under salt treatment compared to the control (Fig. 7b). SlAlba6 was induced by salt stress; its transcript levels did not significantly change during the early phases of salt treatment but gradually increased and peaked (2-fold vs. the control) at $24 \mathrm{~h}$ of exposure to salt stress. Several Alba genes, SlAlba2, SlAlba3, SlAlba5, and SlAlba8, displayed reduced transcript levels (1.5-to 4-fold lower than the control) at one or two time points during salt-stress treatment.

Seven Alba genes were differentially regulated in response to low temperature stress (Fig. 7c). SlAlba8 was significantly upregulated (1.7- to 2-fold vs. the control) by cold treatment, while multiple Alba genes (SlAlba2, SlAlba3, SlAlba4, SlAlba5, SlAlba6, and SlAlba7) were downregulated (1.6- to 12-fold relative to the control) in response to cold stress.

The expression of SlAlba3 was remarkably induced at $24 \mathrm{~h}$ after withholding watering for drought treatment, whereas most Alba genes were significantly deregulated (1.4- to 54-fold vs. the control) at one or multiple time points during the drought stress period (Fig. 7d). The SlAlba genes also showed similar expression patterns in response to the stress hormone ABA. Most of the genes were considerably upregulated (1.8- to 5 -fold higher than the control) under ABA treatment; only one gene, SlAlba3, was unresponsive to ABA treatment (Fig. 7e).

\section{Subcellular location analysis of tomato Alba proteins}

In silico subcellular location analysis suggested that the SlAlba proteins are localized to different parts of the cell, such as the cytoplasm, chloroplasts, and nucleus (Table S11). To verify their predicted localizations, three Alba genes (SlAlba4, SlAlba5, and SlAlba6) were used to generate fusion proteins with GFP. These proteins were expressed in rice protoplasts and their subcellular locations were examined. SlAlba4 was predominantly located in the cytosol, but could also be observed in the nucleus. SlAlba5 was primarily located in the cytosol, with strong signals detected in a large portion of the cytoplasm. SlAlba6 was detected in the nucleus as well as the cytoplasm (Fig. 8).

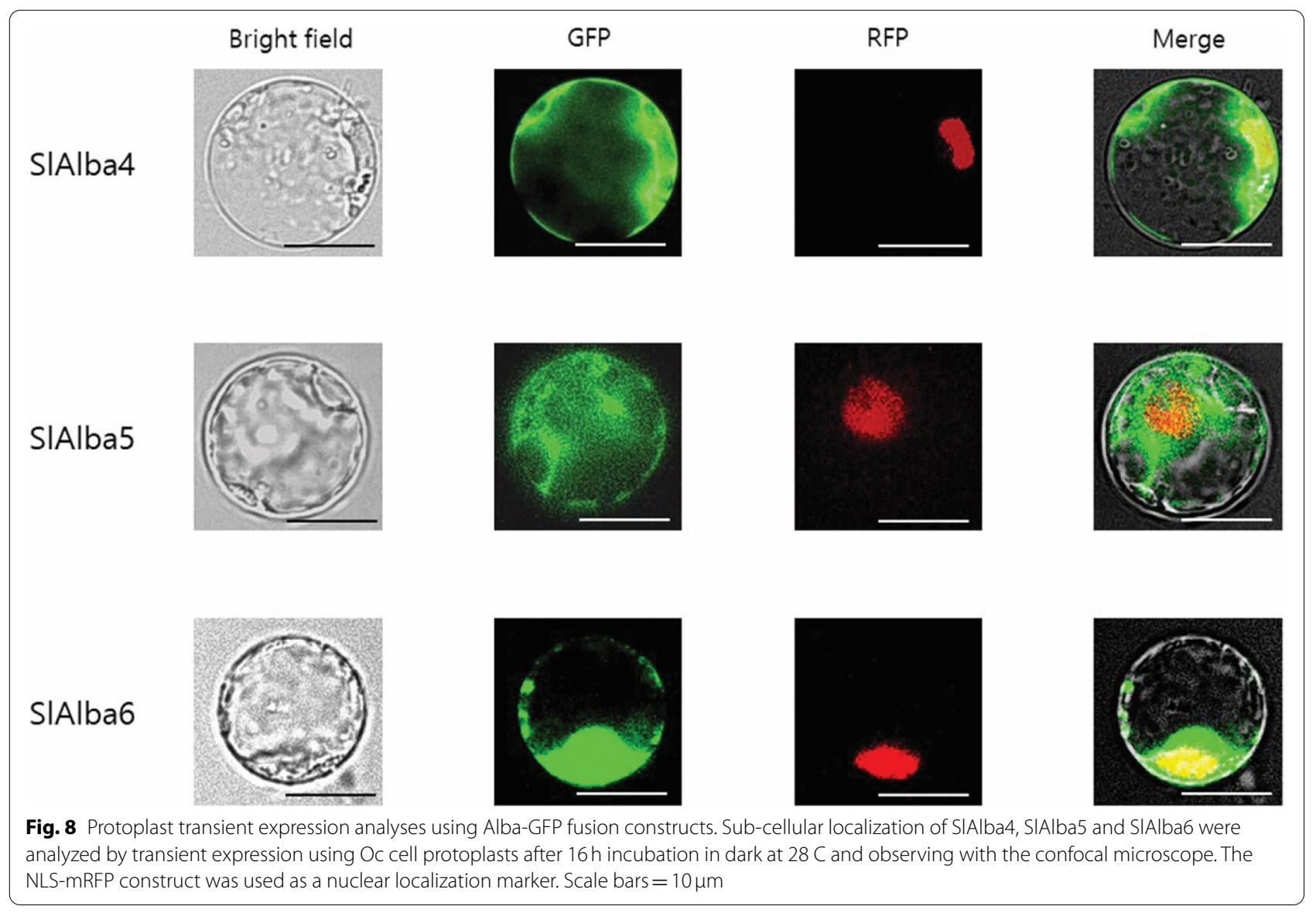




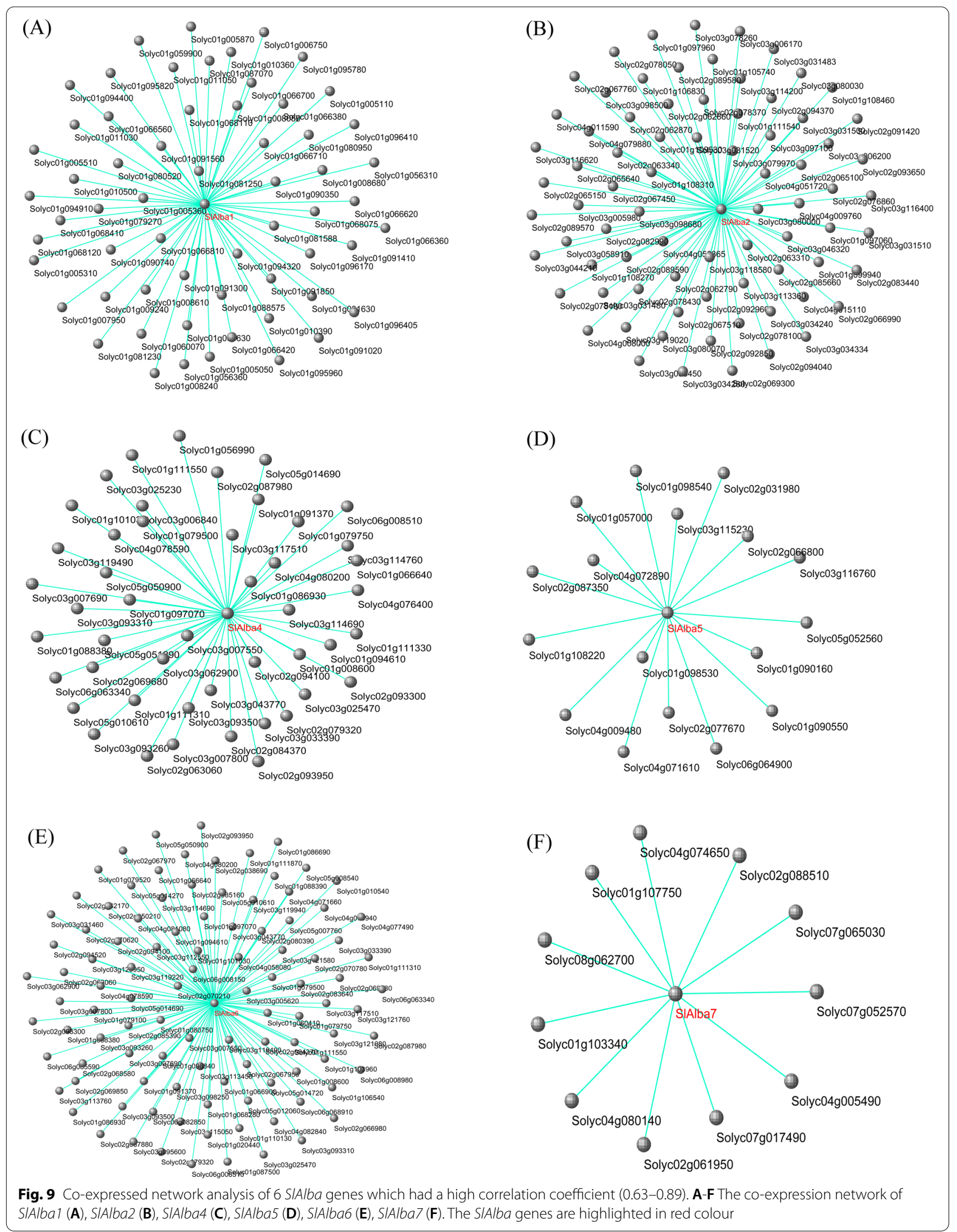




\section{Weighted gene co-expression network analysis} and functional enrichment analysis

The co-expression profiles of SlAlba genes in 18 RNAseq data were analyzed using WGCNA to identify the relationship between the genes. A total of 315 genes were co-expressed with SlAlba genes, and the correlation coefficient ranged from 0.63-0.89 (Fig. 9). In details, we found that $60,77,47,17,103$, and 11 genes were coexpressed with SlAlba1, SlAlba2, SlAlba4, SlAlba5, SlAlba6, and SlAlba7, respectively. However, we could not detect the co-expressed genes of SlAlba3 and SlAlba8 because they were low expressed in selected RNA-seq data.

The GO enrichment and KEGG enrichment analysis of the co-expressed genes showed that most of the genes identified in the co-expression network of SlAlba genes were involved in various biological processes, such as environmental adaptation, starch and sucrose metabolism, transport, signalling and cellular processes, plant-pathogen interaction, pentose and glucuronate interconversions, membrane trafficking, lipid metabolism, lipid biosynthesis proteins, glycerophospholipid metabolism, glycerolipid metabolism, galactose metabolism, flavone and flavonol biosynthesis, exosome, endocytosis, cytoskeleton proteins, carbohydrate metabolism, amino sugar and nucleotide sugar metabolism, pollen tube growth, unidimensional cell growth, cell wall organization or biogenesis, actin filament bundle organization, etc., while some of them have not been assigned to any biological pathway (Fig. 10, Fig. S8 and Table S13). Interestingly, a flower-specific gene SlPLIM2a (Solyc01g094320) in the gene co-expression network of SlAlba1 [30], SlCML8 (Solyc03g097100) and SlCML27 (Solyc02g063340), which are related to environmental adaptation and highly expressed in floral buds, in that of SlAlba2 [31], a heat-inducible gene SIDEAH14 (Solyc05g014690) in that of SlAlba4 [32], a heat-inducible gene HSP101 (Solyc03g115230) in that of SlAlba5 [33], a salt-inducible auxin-related gene $L A X 2$ (Solyc01g111310) and a heat-responsive gene SIDEAH14 (Solyc05g014690) in that of SlAlba6 [32, 34], and a salt-inducible gene SlSYP51.2 (Solyc07g065030) in that of SlAlba7 [35] were also identified, respectively.

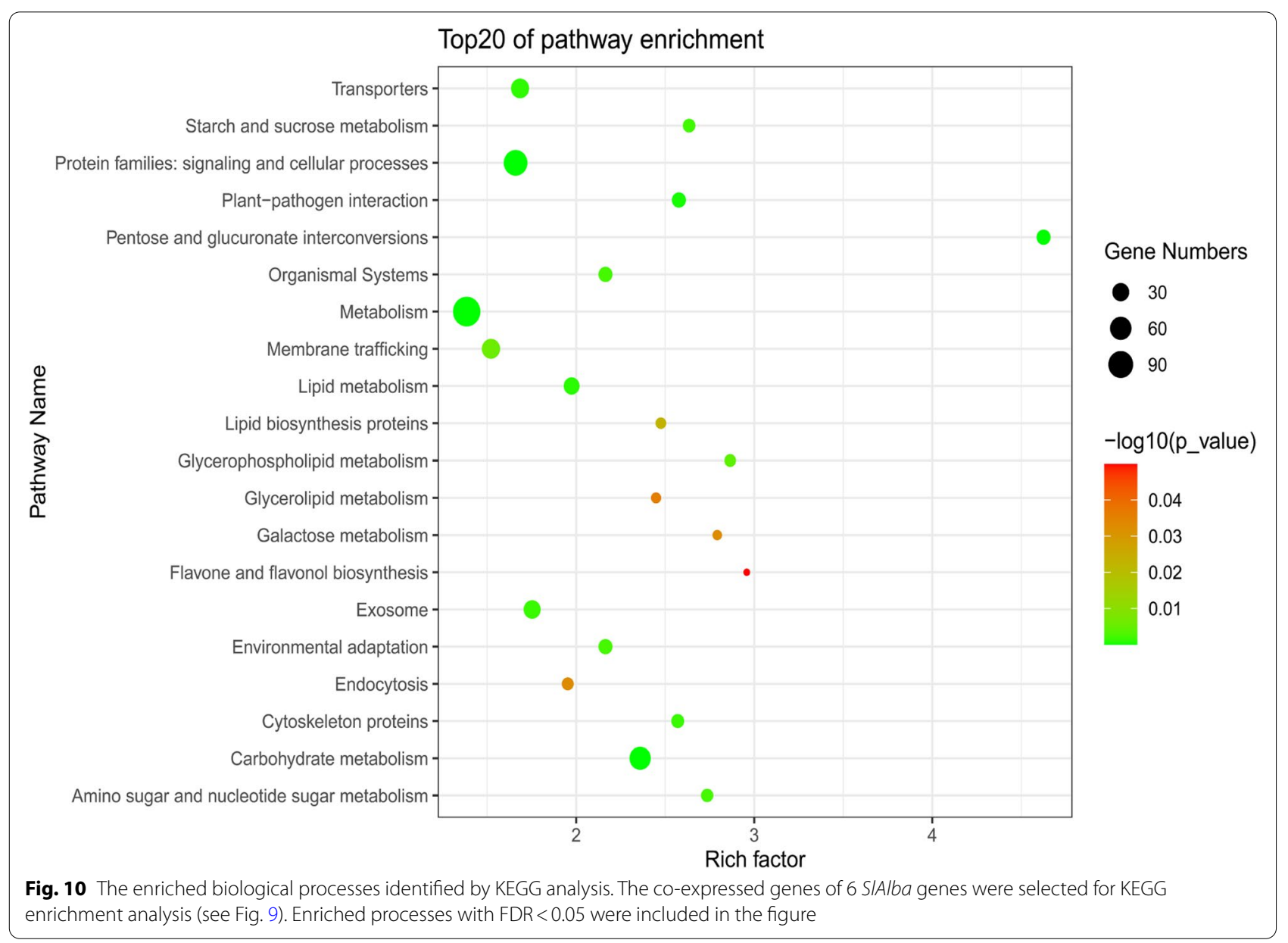




\section{Discussion}

The Alba gene family has been found in almost all kingdoms of life, including archaebacteria [1, 2], suggesting that these genes may have evolved before the divergence of archaea and eukaryotes. Genome-wide identification and comprehensive characterization of Alba gene family has been reported in some plant species, but the tomato Alba gene family has yet to be described. In this study, we identified eight Alba genes from tomato via genome-wide analysis. The number of Alba gene family members was observed to vary across diverse species of the plant kingdom (Fig. 1). Alba genes exist as a multi-gene family in tomato with the average number compared with other plant species, implying that they have biological importance in this plant species.

An in-depth analysis of the phylogenetic relationships among Alba proteins revealed two types. Some of the proteins are relatively large, with an RGG/RG repeat motif or other domain in addition to the generic single Alba domain; most of these proteins belong to the RPP25-like family. The other Alba proteins are relatively small, with only the generic single Alba domain; most of these proteins belong to the RPP20-like family in plants or the archaeal Alba family (Fig. 1). These findings suggest that the emergence of additional motifs and domains in Alba family proteins may have played a significant role in the expansion and diversification of the Alba gene family in plant species during the course of evolution.

Our phylogenetic analysis grouped the plant Alba genes into two distinct subclusters: Alba genes from monocots (rice, sorghum, and maize) and those from dicots (tomato, potato, Arabidopsis, chickpea, and grapevine). In addition, the only Alba gene (CreAlba1) identified in the genome of the single-celled green alga Chlamydomonas reinhardtii was clustered together with those of land plant species in the Rpp20-like phylogenetic group, indicating that common Alba genes are shared by chlorophytes and streptophytes, which diverged over 1 billion years ago [36]. Additionally, Alba genes from the moss Physcomitrella patens, the basal angiosperm Amborella, monocots (sorghum, rice, and maize), and dicots (tomato, potato, grapevine, Arabidopsis, and chickpea) were present within both eukaryotic-specific phylogenetic groups (Fig. 1), suggesting that these two families evolved before bryophytes and angiosperms diverged approximately 450 million years ago [37]. Overall, these findings enabled us to retrace ancient evolutionary transitions in this gene family.

From an evolutionary standpoint, gene duplication events increase the number of genes in a particular gene family, which can help plants adapt to adverse environmental stresses [38, 39]. One duplicated gene pair,
SlAlba6/SlAlba7, was predicted in the tomato genome (Fig. S4), indicating that the eight SlAlba genes appear to have been derived from an original set of seven ancestral genes. Microsynteny analysis revealed three segmentally duplicated gene pairs between tomato and Arabidopsis, but only a single duplicated gene pair between tomato and rice as well as between Arabidopsis and rice. These results are consistent with the closer evolutionary relationship between tomato and the dicotyledonous model plant Arabidopsis than between tomato and the monocotyledonous model plant rice (Fig. 4).

Analysis of genetic structural diversity is indispensable for the evolutionary analysis of a multi-gene family. Detailed analyses of the conserved motifs and exon-intron structures of the Alba genes revealed that exons and introns as well as conserved motifs were organized in a similar pattern among phylogenetically closely related Alba genes but in a different manner among those from the different clusters. These findings point to the functional redundancy across phylogenetically closely related Alba family genes and present a likely rationale for the functional divergence among the divergent Alba genes during the evolutionary process (Figs. S1 and S2).

The predicted three-dimensional structure of a protein provides valuable information about its possible molecular functions and ligand-binding sites. To gain insight into further understanding of the molecular functions of SlAlba proteins, we predicted the 3D models of tomato Alba proteins. A previous study uncovered the likely binding affinity of rice Alba proteins to several molecules including DNA and RNA. In the current study, 3D-modeling of SlAlba proteins also predicted possible binding interactions with DNA, RNA, and peptide molecules (Fig. S6), supporting their putative functions in transcriptional and translational regulation (Table S12).

The proper transport of a protein to its specific subcellular locations is critical for its optimal activity. A previous subcellular localization experiment revealed that OsAlba1 localizes to the nucleus and cytoplasm [40]. In support of this finding, tomato Alba fusion proteins also showed GFP signals in the nucleus and cytoplasm (Fig. 8). These findings suggest that the diverse Alba proteins are involved in a variety of cellular signalling processes in the cytoplasm and nucleus.

Analyzing the expression patterns of a gene during growth and development and upon exposure to stress stimuli may help determine its functions. Thus, we analyzed the expression profiling of SlAlba genes in various organs and under different abiotic stresses. The majority of SlAlba genes except SlAlba1 exhibited differential expression profiles across the organs examined, suggesting these genes play distinct regulatory roles in growth 
and development (Fig. 6). Several of the tomato Alba genes (SlAlba3, SlAlba4, SlAlba6, SlAlba7, and SlAlba8) were predominantly expressed in vegetative organs, but others, including SlAlba1, SlAlba2, and SlAlba5, showed higher transcript levels in reproductive organs such as flowers and fruits. These results suggest that these genes play preferential roles in these organs and developmental phases in tomato.

The initiation and development of a floral bud, a process modulated by multiple floral genes and environmental factors, plays a vital role in fruit set and crop yield [41]. Intriguingly, SlAlba1 was expressed at strikingly higher levels in flower buds, fully bloomed flowers, and senescent flowers compared to root tissues and $1 \mathrm{~cm}$ fruits, and no expression was detected in other organs. SlAlba2 was predominantly expressed in flower buds, with its expression level many times higher than that in any other organs (Fig. 6). These findings suggest that SlA$l b a 2$ regulates floral bud formation in tomato and that SlAlba1 regulates flower development at all stages via interactions with other regulatory genes.

The roles of Alba genes in fruit development have not previously been studied in any vegetable crop. Tomato is a model organism for the study of climacteric fruits. Therefore, the molecular pathways controlling tomato fruit enlargement (which includes a cell division stage and a cell elongation stage) as well as fruit ripening have been extensively explored [42-44]. Interestingly, SlAlba5 is the only gene that showed higher expression in tomato fruits at all stages of development (except the immature stage), with its peak expression in $1 \mathrm{~cm}$ fruits. By contrast, the remaining seven SlAlba genes showed lower expression levels in fruits at all developmental stages compared to the control (leaves; Fig. 6). Our findings suggest that SlAlba5 plays a regulatory role in fruit enlargement and ripening, particularly during the initial stages of fruit development.

Most tomato Alba genes that were highly expressed in vegetative organs had their highest expression levels in stems; only one gene had its highest expression level in leaves, and no gene has its highest expression level in roots. In addition to providing mechanical support to the aerial portions of the plant, the stem facilitates the longdistance translocation of water and nutrients to sustain plant growth under both normal and stressful conditions. The transcript levels of SlAlba3, SlAlba4, SlAlba6, SlAlba7, and SlAlba8 were markedly higher in stems than in any other organ examined, suggesting that they likely function in stem growth, the long-range movement of water and nutrients, and stress tolerance. The higher expression level of SlAlba3 in leaves suggests that it might play a role in leaf development and signalling cascades in leaves (Fig. 6). Taken together, these findings point to the diverse functions of SlAlba family genes in plant development.

Plant responses and adaptation to environmental stresses often involve differential gene expression, which is regulated by a dynamic network of numerous transcription factors and various stress tolerance genes in an ABA-dependent or -independent manner [45-48]. Alba proteins are associated with stress tolerance due to their involvement in genome packaging and organization, transcriptional and translational regulation, post-translational regulation, and RNA metabolism, in addition to responses to different environmental stresses [2, 22, 49]. The possible roles of Alba family genes in plant stress responses were supported by previous studies reporting the stress-induced expression of Alba genes in several plant species, such as cotton, Arabidopsis, and rice [15, $22,23]$. The role of OsAlba1 as a DNA binding protein involved in oxidative stress tolerance was revealed by complementation analysis in yeast. Moreover, the susceptibility of ghAlba4- and ghAlba5-silenced cotton plants to drought and salinity conditions highlighted the possible involvement of Alba genes in plant stress tolerance [23, 40].

We also observed that tomato Alba genes were differentially expressed in response to various abiotic stresses (Fig. 7a-e). SlAlba4 and SlAlba5 expression was significantly induced by heat treatment (Fig. 7a), which is in agreement with the finding that many Alba family genes in Arabidopsis and rice were markedly upregulated in response to mild heat stress $\left(37^{\circ} \mathrm{C}\right)$ and moderate heat stress $\left(42^{\circ} \mathrm{C}\right)$, respectively $[15,22]$. Here we showed that SlAlba6 was sharply upregulated in plants under saline conditions (Fig. 7b), suggesting its possible role in salt tolerance in tomato. This result is in agreement with the recent study finding that Alba genes were expressed at higher levels in cotton upon exposure to salt stress and that ghAlba4 and ghAlba5 cotton plants showed increased sensitivity to salt treatment compared to wild type and control plants [23]. The upregulation of SlAlba3 at $24 \mathrm{~h}$ after withholding watering revealed its probable involvement in response to drought stress. This finding is supported by previous reports in which several Alba genes in rice and cotton were upregulated by dehydration treatment $[22,23]$. The expression level of SlAlba8 was considerably elevated following cold stress (Fig. 7c), which is consistent with the finding that a few rice Alba genes were upregulated in response to low temperature stress [22]. The phytohormone ABA is well known for its role in regulating plant acclimation to adverse environmental stresses, including heat, cold, salt, and drought [50-52]. All tomato Alba genes except SlAlba3 were markedly upregulated under ABA treatment (Fig. 7e), which agrees with the finding that multiple Alba genes in 
rice were induced by ABA treatment [22]. Therefore, SlA$l b a$ genes might function in abiotic stress tolerance via their direct roles in ABA signalling.

In order to better understand the probable roles of SlAlba genes under various abiotic stresses, we analyzed the prevalence of the cis-acting elements in the upstream regions of tomato Alba genes. The regulation of tomato Alba genes under stress conditions was also corroborated by the prevalence of multiple cis-elements related to stress tolerance and hormonal responses in their promoter regions. Such elements might facilitate the regulation of these genes under different abiotic stress conditions (Fig. S5, Table S5).

Post-transcriptional regulation of numerous miRNA families plays a vital role in various biological processes, including development and stress tolerance [53, 54]. Several target sites for miRNAs related to stress tolerance and developmental processes were predicted in SlAlba genes (Table S6). This result is consistent with the finding that miRNA target sites are present in the Alba genes of various plant species including rice, Arabidopsis, maize, and sorghum [22].

To shed light on the putative functional roles of SlAlba genes in tomato, a gene co-expression network was constructed using the RNA-seq data. Multiple genes involved in several biological pathways showed co-expression with most SlAlba genes (Figs.9 and 10, Fig. S8, and Table S13), highlighting the biological importance of SlAlba gene family in tomato. In addition to co-expression with many genes related to several biological pathways, the floral-specific gene SlAlba1 was co-expressed with a transcription factor SlPLIM2a (Solyc01g094320), which shows flower specific expression in tomato [30], suggesting that they might function together in the flower development of tomato [REF]. SlAlba2, which is highly expressed in floral buds, displayed co-expression with several genes related to plant-pathogen interaction and environmental adaptation (Table S13). In addition, it was co-expressed with SlCML8 (Solyc03g097100) and SlCML27 (Solyc02g063340), which are predominantly expressed in flower buds [31]. These findings corroborated the role of SlAlba2 in abiotic stress response and flower bud development in tomato. SlAlba4 and SlA$l b a 5$, which were upregulated by heat treatment, were also co-expressed with heat-inducible genes SIDEAH14 (Solyc05g014690) and HSP101 (Solyc03g115230), respectively, implying that they might have interaction for the adaptation of tomato to heat stress [32, 33]. Similarly, SlAlba6, which is upregulated by salt stress, showed co-expression with a salinity induced auxin-related gene LAX2 (Solyc01g111310) [34], hinting that they might interact with each other for the improvement of salt tolerance in tomato. SlAlba 7 was co-expressed with a salt-inducible gene SISYP51.2 (Solyc07g065030) [35], a gene involved in environmental information processing (Solyc01g107750) and the genes related to chaperones and folding catalysts (Solyc02g061950 and Solyc08g062700) (Table S13), suggesting its important role in the abiotic stress response of tomato. Taken together, the findings of this study provide a valuable resource for further analysis to explore the biological functions of Alba proteins in tomato.

\section{Conclusions}

In the current study, we identified eight SlAlba genes in tomato. These genes were clustered into two phylogenetic groups based on their domain and motif architectures. The likely involvement of these SlAlba genes in diverse signalling pathways, developmental cascades, and plant stress responses is underscored by the presence of development- and stress response-associated cis-regulatory elements and miRNA target sites in their sequences, their dual localization to the cytoplasm and nucleus, and their putative binding to several ligands including DNA, RNA, and peptides. Expression profiling revealed that most SlAlba genes are differentially expressed across various organs and in response to different stimuli. The expression pattern of SlAlba5 suggests that it functions in fruit enlargement and ripening, especially during the cell division phase. Several SlAlba genes were significantly induced under different abiotic stresses, such as SlAlba4 and SlAlba5 by heat, SlAlba6 by salt, SlAlba 8 by cold stress, and SlAlba3 by drought stress, pointing to their possible roles in tolerance to these stresses. Co-expression network analysis highlighted the biological importance of SlAlba genes in tomato and the identified co-expressed genes with SlA$l b a$ genes supported their probable roles in the development and abiotic stress response of tomato. Our findings lay the foundation for further exploring the SlAlba gene family and provide new perspectives for the genetic improvement of tomato.

\section{Methods \\ In silico identification and sequence analysis of tomato Alba genes}

We identified tomato Alba gene family members from the Phytozome database (http://www.phytozome.net) using the keyword "Alba" [55] and performed BLAST searches of the Sol genomics database (http://www. solgenomics.net/tools/blast/) [56] using Arabidopsis thaliana Alba protein sequences obtained from TAIR (https://www.Arabidopsis.org/) as queries [57]. To 
validate the presence of the Alba domain, the resulting eight non-redundant Alba protein sequences were subjected to searches using the NCBI CDD search (https:// www.ncbi.nlm.nih.gov/Structure/bwrpsb/bwrpsb.cgi) [58] and the SMART web tool (http://smart.emblheidel berg.de/) [59]. The exon/intron structures were visualized using the Gene Structure Display Server-2.0 web server (http://gsds.cbi.pku.edu.cn/index.php) by loading both SlAlba genomic and coding sequences [60]. The protein length (the number of amino acids), molecular weight, GRAVY values (grand average of hydropathicity index), and isoelectric points of the SlAlba proteins were determined using ProtParam (http://cn.expasy.org/ tools/protparam.html) [61]. The Open Reading Frame Finder tool (https://www.ncbi.nlm.nih.gov/orffinder/) was used to analyze the open reading frames of the $S l A$ lba genes. Clustal Omega (Clustal Omega < Multiple Sequence Alignment < EMBL-EBI) and ESPript web server (https://espript.ibcp.fr/ESPript/cgi-bin/ESPri pt.cgi) were used for multiple-protein sequence alignment $[62,63]$. The web tool "Immunomedicine Group" (http://imed.med.ucm.es/Tools/sias.html) was employed to analyze the sequence homology of the eight Alba proteins. The MEME suite motif search tool (http://memes uite.org/) was used to examine the conserved motifs in protein sequences with the following parameters: maximum number of motifs 15 and motif length between six and 50 amino acids [64]. WoLF-PSORT (https://wolfp sort.hgc.jp/) was used to predict the subcellular localizations of the SlAlba proteins [65].

\section{Phylogenetic analysis}

The full-length Alba protein sequences retrieved from the Phytozome and NCBI databases were aligned using Clustal Omega, followed by phylogenetic analysis via the neighbor-joining (NJ) method with 1000 bootstrap replications in MEGA 6.0 [66]. The names of the genes used to build the phylogenetic tree, together with their accession numbers, are described in Table S1.

\section{Prediction of miRNA target sites, cis-regulatory elements, and chromosomal locations}

The putative miRNA targets were analyzed using the psRNATarget web tool (http://plantgrn.noble.org/psRNA Target/analysis). The PlantCare database (http://bioin formatics.psb.ugent.be/webtools/plantcare/html/) was employed to investigate the promoter region of each gene $1500 \mathrm{bp}$ upstream of the initiation codon [ATG] to predict putative cis-acting elements in these promoters. The SlAlba genes were mapped to chromosomes using the online tool MapGene2Chrom web v2 (http://mg2c.iask. in/mg2c_v2.0/) after determining their chromosomal positions from the Sol genomic database.

\section{Gene duplication and microsynteny analysis}

Gene duplications events across tomato Alba genes were identified with the one-step MCScanX program of TBtools software [67] and checked by BLASTP with the E-value $<10^{-10}$. The identified duplicated gene pairs were visualized in Advanced Circos. The synonymous (Ks) and non-synonymous (Ka) nucleotide substitution rates of the SlAlba genes were computed following the method of Nei and Gojobori [68]. The $\mathrm{Ka} / \mathrm{Ks}$ ratio was analyzed to determine the mode of selection [69]. The formula $\mathrm{T}=\mathrm{Ks} / 2 \mathrm{r}$ MYA (millions of years ago) was used to estimate the divergence time $(\mathrm{T})$ for the duplicated gene pairs. Ks is the synonymous substitution rate per site, and $\mathrm{r}$ is the constant for dicot plants of $1.5 \times 10^{-8}$ substitutions per site per year [70]. Using a reciprocal BLAST search against the entire genomes of tomato, Arabidopsis, and rice, the microsyntenic relationship of Alba genes across these species was analyzed. The TBtools software was used to visualize the duplicated gene pairs [67].

\section{Comparative modelling of tomato Alba proteins}

The 3D structures of the SlAlba proteins were predicted with the I-TASSER server using the protein sequences of SlAlba1-8 as input [71]. 3D models were generated by multiple threading of the alignments with LOMETS and performing iterative TASSER assembly simulations. The best-modelled structures with the maximum scores were selected and the template analogues were also identified. The resulting molecular models were refined with ModRefiner [72]. The functions of the modelled proteins were predicted using the I-TASSER server based on global and local similarity to template proteins in PDB with known structures and functions.

\section{Subcellular localization}

The tomato Alba cDNA was amplified using gene-specific primers (Table S2) and cloned into the pGA3452 vector harbouring the maize $\mathrm{Ubi1}$ promoter, the synthetic GFP coding region, and the nos terminator [73]. NLS-mRFP under the control of the $35 \mathrm{~S}$ promoter was employed as a nuclear marker. The constructs were cotransformed via electroporation into protoplasts prepared from rice Oc cells, and the resulting transformants were incubated overnight at $28^{\circ} \mathrm{C}$ in the dark for 12 to $16 \mathrm{~h}$. Fluorescent signals were visualized under a filter-equipped microscope (BX61; Olympus, Tokyo, Japan) using bright field illumination, the GFP channel, and the red fluorescent protein channel.

\section{Preparation of plant materials and stress treatments}

Tomato plants (Solanum lycopersicum cv. Ailsa Craig) were grown in soil in a growth room under controlled 
conditions at a temperature of $25^{\circ} \mathrm{C}$ during the day and $20^{\circ} \mathrm{C}$ at night and a $16 \mathrm{~h}$ light $8 \mathrm{~h}$ dark photoperiod. For tissue-specific expression analysis, fresh roots, stems, and leaves were collected from 28-day-old plants. The remaining plants were transferred to a greenhouse maintained at $25 / 20^{\circ} \mathrm{C}$ day/night temperatures until the reproductive stage to collect flower and fruit samples. Three different types of flower samples were harvested: floral buds, full-blooming flowers, and senescent flowers. Fruit samples were collected at six different stages: (i) young fruits approximately $1 \mathrm{~cm}$ in diameter at 2 weeks after pollination ( $1 \mathrm{~cm}$ fruits) (ii) immature fruits at 5 weeks after pollination (IM fruits), (iii) mature green fruits at 7 weeks after pollination (MG fruits), (iv) breaker fruits, when the colour of mature fruits changes from green to faint yellow-orange (B fruits), (v) breaker after 3 days (B3 fruits), and (vi) breaker after 7 days (B7 fruits) [74].

To study expression patterns of SlAlba genes under different abiotic stress conditions (abscisic acid [ABA], heat, cold, and salt $[\mathrm{NaCl}])$, leaf samples from 28-day-old seedlings of uniform growth and development were collected at $0,1,3,9$, and $24 \mathrm{~h}$ after the start of the stress treatments [74], while they were collected at $0,24,48,72$, and $96 \mathrm{~h}$ after withholding watering for drought treatment. ABA treatment was applied by spraying leaves with $100 \mu \mathrm{M}$ ABA. To impose heat and cold stress, the plants were incubated in a growth cabinet at $40^{\circ} \mathrm{C}$ and $4{ }^{\circ} \mathrm{C}$, respectively. Salt stress was imposed by submerging roots in a $200 \mathrm{mM} \mathrm{NaCl}$ solution. Drought treatment was applied to the plants by withholding watering after watering until the soil mixture is sufficiently wet. Plants grown in soil under normal conditions $\left(25^{\circ} \mathrm{C}\right)$ were used as the $0 \mathrm{~h}$ controls for all stress treatments. The samples were collected from three biological replicates, immediately frozen in liquid nitrogen, and stored at $-80^{\circ} \mathrm{C}$ for RNA isolation.

\section{RNA extraction and qPCR expression analysis}

Total RNA was isolated from the samples using an RNeasy Mini kit (Qiagen, Hilden, Germany) and purified with an RNase-free DNase I kit (Qiagen, Hilden, Germany) in accordance with the manufacturer's protocols. RNA concentrations were determined with a NanoDrop ${ }^{\circledR} 1000$ spectrophotometer (Wilmington, DE, USA), and $1 \mu \mathrm{g}$ total RNA was used to synthesize cDNA using a Superscript ${ }^{\circledR}$ III First-Strand cDNA synthesis kit (Invitrogen, Carlsbad, CA, USA) as per the manufacturer's instructions. Primer3 software (http://frodo. wi.mit.edu/primer3/input.htm) was used to design the gene-specific primers for all tomato Alba genes (Table S3). Melting curve analysis was performed to verify the specificity of the amplicon for each primer pair [22], and the expression of 18S rRNA (F: AAA AGGTCGACGCGGGCT, R: CGACAGAAGGGACGA GAC) was used as an internal control for normalization [75]. qRT-PCR was performed in a $10 \mu \mathrm{L}$ reaction mixture consisting of $1 \mu \mathrm{L}(50 \mathrm{ng}) \mathrm{cDNA}, 2 \mu \mathrm{L}$ forward and reverse primers ( 5 pmol concentration), $5 \mu \mathrm{L}$ of iTaq SYBR Green (Qiagen, Hilden, Germany), and $2 \mu \mathrm{L}$ double distilled water. A Light cycler $^{\circledR}$ 96SW 1.1 (Roche, Germany) was used to amplify and record the Cq value of each sample with the following conditions: predenaturation at $95^{\circ} \mathrm{C}$ for $5 \mathrm{~min}$ followed by 40 cycles at $94^{\circ} \mathrm{C}$ for $10 \mathrm{~s}$, annealing at $58^{\circ} \mathrm{C}$ for $10 \mathrm{~s}$, and extension at $72^{\circ} \mathrm{C}$ for $15 \mathrm{~s}$. The $2^{-\Delta \Delta \mathrm{Ct}}$ method was used to determine the relative transcript levels of each gene against each treatment [76].

\section{Statistical analysis}

Data were analyzed with SigmaPlot 14 (SYSTAT and MYSTAT Products, United States, and Canada) using two-tailed Student's t-tests. ${ }^{*} P \leq 0.05$ and ${ }^{* *} P \leq 0.01$ and ${ }^{* * * *} P \leq 0.001$ were considered statistically significant.

\section{Co-expression network analysis of SIAlba genes}

Raw RNA-Seq data of 18 experiments with 3 repeats were downloaded from the NCBI SRA database under the accessions PRJNA639840, PRJNA484882, PRJNA756379, and PRJNA655574 (Table S14). Raw sequence reads were assessed with the FastQC toolkit [77]. The adapter contaminations and low-quality reads were removed using FASTP 0.19.4 with the parameters: -q $20-\mathrm{u} 40-\mathrm{n} 10-150$ [78]. The clean reads were mapped on tomato reference genome ITAG4.0 by using HISAT2 2.1.0 [79]. FeatureCounts 1.6.2 was used to count the number of reads that mapped on exons [80]. Further analyses were carried out based on fragments per kilo-base per million fragments mapped (FPKM) values. removeBatchEffect processing was performed to remove batch effect in $R$ [81]. Weighted gene coexpression network analysis (WGCNA) was used to search for the co-expression genes with SlAlba genes. The genes with FPKM less than 1 were filtered out, and the remaining genes were input into the WGCNA v1.69 package in $\mathrm{R}$ for co-expression network construction [82]. Pearson correlation matrix and network topology analysis were used to calculate the gene correlation and soft thresholding power, respectively. The power, minModuleSize, and mergeCutHeight value were set to 16 , 30 , and 0.25 , respectively. The networks were visualized using Cytoscape v3.8.2. The GO and KEGG annotations of co-expressed genes with SlAlba genes were conducted by pannzer2 [83] and KEGG Automatic Annotation Server [84]. 


\section{Abbreviations}

Alba: Acetylation lowers binding affinity; 3D: Three Dimensional; GFP: Green fluorescent protein; IF3-C: Translation initiation factor 3 carboxy-terminal; Sir2: Sirtuin 2; Rpp20: Ribonuclease P protein subunit p20; Rpp25: Ribonuclease P protein subunit p25; NAD ${ }^{+}$: Nicotinamide adenine dinucleotide; RGG/ RG: Glycine-arginine-rich; SnRNP : Small nuclear ribonucleoproteins; CLIP1: CAP-Gly domain-containing linker protein 1; ATP: Adenosine triphosphate; TM: Template modeling; RMSD: Root-mean-square deviation; C-score: Confident score; ABA: Abscisic acid; WGCNA: Weighted gene co-expression network analysis.

\section{Supplementary Information}

The online version contains supplementary material available at https://doi. org/10.1186/s12870-021-03310-0.

Additional file 1: Figure S1. Schematic representation of the exon-intron distribution of SIAlba gene family. Figure S2. Schematic representation of 15 conserved motifs in Alba proteins from tomato, Arabidopsis, and rice as predicted by Multiple Em for Motif Elicitation (MEME) web server. Figure S3. Chromosome distribution of tomato Alba genes. Figure S4. Gene duplication investigation of Alba genes in the tomato genome. Figure S5. Putative cis-acting elements in the upstream of SIAlba genes. Figure S6. The predicted binding of putative patterner ligands to SIAlba proteins. Figure S7. Overview of conserved motifs of Alba proteins from tomato, Arabidopsis and rice determined using MEME web tool. Figure S8. The top 30 enriched $\mathrm{GO}$ terms of co-expressed genes with 6 SIAlba genes. Table S1. List of the Alba amino acid sequences used for phylogenetic investigation. Table S2. The primer sequences used for subcellular localization analysis. Table S3. The primer sequences of SIAlba genes used for qRT-PCR analysis. Table S4. Sequence identity among 8 tomato Alba proteins. Table S5. List of cis-elements in the promoter regions tomato Alba genes. Table S6. Prediction of miRNA target sequences in tomato Alba genes. Table S7. Templates used for 3D structure modelling of SIAlba proteins. Table S8. Secondary structural components in SIAlba proteins. Table S9. Secondary structure prediction for SIAlba proteins by I-TASSER. Table S10. Parameters for 3D structure modelling of SIAlba proteins. Table S11. Subcellular localization of SIAlba proteins predicted by in silico analysis. Table S12. Gene Ontology (GO) annotation for SIAlba proteins. Table S13. Annotated pathways of co-expressed genes. Table S14. Information of samples used for RNA seq analysis.

\section{Acknowledgements}

Not applicable.

\section{Authors' contributions}

D.-J.L., J.-M.L., C.-K.K. and M.-Y.C. conceived the study and design the work. M.-Y.C., D.-J.L., J.-M.L. and C.-K.K. supervised and monitored the experimental study; A.H.W., X.P and M.W. carried out in silico analysis. A.H.W. grew plants, isolated RNA, conducted expression analysis, analyzed the data and write the original draft following the guidance of M.Y.C.L.H.C performed the subcellular localization experiments. All authors made contributions to the manuscript. The author(s) read and approved the final manuscript.

\section{Funding}

This research was supported by the National Research Foundation of Korea (NRF) Grant funded by the Korean government (MSIT) (no. NRF-2018R1A2B6001781).

\section{Availability of data and materials}

We declare that the dataset(s) required to reproduce the results of this article are included in the article and additional file(s) available in the journal webpage.

\section{Declarations}

Ethics approval and consent to participate Not Applicable.
Consent for publication

Not applicable.

\section{Competing interests}

The authors declare that they have no competing interests.

\section{Author details}

'Department of Agricultural Education, Sunchon National University, 413 Jungangno, Suncheon, Jeonnam 540-950, Republic of Korea. ${ }^{2}$ Department of Biology, Yangon University of Education, Kamayut Township, Yangon Region 11041, Myanmar. ${ }^{3}$ Department of Plant Bioscience, College of Natural Resources and Life Science, Pusan National University, Miryang-si, Gyeongsangnam-do 50463, Republic of Korea. ${ }^{4}$ Institution of Genomics and Bioinformatics, South China Agricultural University, Guangzhou, China. ${ }^{5}$ College of horticulture, South China Agricultural University, Guangzhou, China. ${ }^{6}$ Department of Horticulture, Kyungpook National University, Daegu, Republic of Korea.

Received: 12 July 2021 Accepted: 2 November 2021

Published online: 12 November 2021

\section{References}

1. Aravind $L$, lyer $L M$, Anantharaman $V$. The two faces of Alba: the evolutionary connection between proteins participating in chromatin structure and RNA metabolism. Genome Biol. 2003;4:1-9.

2. Goyal M, Banerjee C, Nag S, Bandyopadhyay U. The Alba protein family: structure and function. Biochim Biophys Acta. 2016;1864:570-83.

3. Črnigoj M, Podlesek Z, Zorko M, Jerala R, Anderluh G, Ulrih NP. Interactions of archaeal chromatin proteins Alba1 and Alba2 with nucleic acids. PLoS One. 2013;8:e58237.

4. Jelinska C, Conroy MJ, Craven CJ, Hounslow AM, Bullough PA, Waltho $J$, et al. Obligate heterodimerization of the archaeal Alba2 protein with Alba1 provides a mechanism for control of DNA packaging. Structure. 2005;13:963-71.

5. Jelinska C, Petrovic-Stojanovska B, Ingledew WJ, White MF. Dimer-dimer stacking interactions are important for nucleic acid binding by the archaeal chromatin protein Alba. Biochem J. 2010;427:49-55.

6. Laurens N, Driessen RP, Heller I, Vorselen D, Noom MC, Hol FJ, et al. Alba shapes the archaeal genome using a delicate balance of bridging and stiffening the DNA. Nat Commun. 2012;3:1-8.

7. Bell SD, Botting CH, Wardleworth BN, Jackson SP, White MF. The interaction of Alba, a conserved archaeal chromatin protein, with Sir2 and its regulation by acetylation. Science. 2002;296:148-51.

8. Marsh VL, Peak-Chew SY, Bell SD. Sir2 and the acetyltransferase, pat, regulate the archaeal chromatin protein, Alba. J Biol Chem. 2005;280:21122-8.

9. White MF, Bell SD. Holding it together: chromatin in the Archaea. Trends Genet. 2002;18:621-6.

10. Wardleworth BN, Russell RJ, Bell SD, Taylor GL, White MF. Structure of Alba: an archaeal chromatin protein modulated by acetylation. EMBO J. 2002;21:4654-62.

11. Zhao K, Chai X, Marmorstein R. Structure of a Sir2 substrate, Alba, reveals a mechanism for deacetylation-induced enhancement of DNA binding. J Biol Chem. 2003;278:26071-7.

12. Forterre P, Confalonieri F, Knapp S. Identification of the gene encoding archeal-specific DNA-binding proteins of the Sac10b family. Mol Microbiol. 1999;32:669-70.

13. Guo R, Xue H, Huang L. Ssh10b, a conserved thermophilic archaeal protein, binds RNA in vivo. Mol Microbiol. 2003;50:1605-15.

14. She Q, Singh RK, Confalonieri F, Zivanovic Y, Allard G, Awayez MJ, et al. The complete genome of the crenarchaeon Sulfolobus solfataricus P2. Proc Natl Acad Sci. 2001;98:7835-40.

15. Náprstková A, Malínská K, Záveská Drábková L, Billey E, Náprstková D, Sýkorová E, et al. Characterization of ALBA family expression and localization in Arabidopsis thaliana generative organs. Int J Mol Sci. 2021;22:1652

16. Thandapani P, O'Connor TR, Bailey TL, Richard S. Defining the RGG/RG motif. Mol Cell. 2013;50:613-23.

17. Dupé A, Dumas C, Papadopoulou B. An Alba-domain protein contributes to the stage-regulated stability of amastin transcripts in Leishmania. Mol Microbiol. 2014;91:548-61. 
18. Gissot M, Walker R, Delhaye S, Alayi TD, Huot L, Hot D, et al. Toxoplasma gondii Alba proteins are involved in translational control of gene expression. J Mol Biol. 2013;425:1287-301.

19. Wai $A H$, Naing AH, Lee DJ, Kim CK, Chung MY. Molecular genetic approaches for enhancing stress tolerance and fruit quality of tomato. Plant Biotechnol Rep. 2020;14:515-537.

20. Dupé A, Dumas C, Papadopoulou B. Differential subcellular localization of Leishmania Alba-domain proteins throughout the parasite development. PLoS One. 2015:10:e0137243.

21. Mani J, Güttinger A, Schimanski B, Heller M, Acosta-Serrano A, Pescher P, et al. Alba-domain proteins of Trypanosoma brucei are cytoplasmic RNA binding proteins that interact with the translation machinery. PLoS One. 2011;6:e22463.

22. Verma JK, Wardhan V, Singh D, Chakraborty S, Chakraborty N. Genomewide identification of the Alba gene family in plants and stress-responsive expression of the rice Alba genes. Genes. 2018;9:183.

23. Magwanga RO, Kirungu JN, Lu P, Cai X, Xu Y, Wang X, et al. Knockdown of ghAlba_4 and ghAlba_5 proteins in cotton inhibits root growth and increases sensitivity to drought and salt stresses. Front Plant Sci. 2019;10:1292

24. Ozdilek BA, Thompson VF, Ahmed NS, White Cl, Batey RT, Schwartz JC. Intrinsically disordered RGG/RG domains mediate degenerate specificity in RNA binding. Nucleic Acids Res. 2017:45:7984-96.

25. Vasilyev N, Polonskaia A, Darnell JC, Darnell RB, Patel DJ, Serganov A. Crystal structure reveals specific recognition of a G-quadruplex RNA by a $\beta$-turn in the RGG motif of FMRP. Proc Natl Acad Sci. 2015;112:E5391-400.

26. López-Galiano MJ, Sentandreu V, Martínez-Ramírez AC, Rausell C, Real MD, Camañes G, et al. Identification of stress associated microRNAs in Solanum lycopersicum by high-throughput sequencing. Genes. 2019;10:475.

27. Liu M, Yu H, Zhao G, Huang Q, Lu Y, Ouyang B. Identification of droughtresponsive microRNAs in tomato using high-throughput sequencing. Funct Integr Genomics. 2018;18:67-78.

28. Tripathi A, Goswami K, Tiwari M, Mukherjee SK, Sanan-Mishra N. Identification and comparative analysis of microRNAs from tomato varieties showing contrasting response to ToLCV infections. Physiol Mol Biol Plants. 2018;24:185-202.

29. Zhang B. MicroRNA: a new target for improving plant tolerance to abiotic stress. J Exp Bot. 2015;66:1749-61.

30. Khatun K, Robin AH, Park Jl, Ahmed NU, Kim CK, Lim KB, et al. Genomewide identification, characterization and expression profiling of LIM family genes in Solanum lycopersicum L. Plant Physiol Biochem. 2016;108:177-90.

31. Munir S, Khan MR, Song J, Munir S, Zhang Y, Ye Z, et al. Genome-wide identification, characterization and expression analysis of calmodulin-like (CML) proteins in tomato (Solanum lycopersicum). Plant Physiol Biochem. 2016;102:167-79.

32. Xu R, Zhang S, Lu L, Cao H, Zheng C. A genome-wide analysis of the RNA helicase gene family in Solanum lycopersicum. Gene. 2013;513:128-40.

33. Ding H, Mo S, Qian Y, Yuan G, Wu X, Ge C. Integrated proteome and transcriptome analyses revealed key factors involved in tomato (Solanum lycopersicum) under high temperature stress. Food Energy Secur. 2020;9:e239.

34. Martínez-Andújar C, Martínez-Pérez A, Ferrández-Ayela A, Albacete A, Martínez-Melgarejo PA, Dodd IC, et al. Impact of overexpression of 9-cisepoxycarotenoid dioxygenase on growth and gene expression under salinity stress. Plant Sci. 2020;295:110268.

35. Salinas-Cornejo J, Madrid-Espinoza J, Ruiz-Lara S. Identification and transcriptional analysis of SNARE vesicle fusion regulators in tomato (Solanum lycopersicum) during plant development and a comparative analysis of the response to salt stress with wild relatives. J Plant Physiol. 2019:242:153018.

36. Merchant SS, Prochnik SE, Vallon O, Harris EH, Karpowicz SJ, Witman GB, et al. The Chlamydomonas genome reveals the evolution of key animal and plant functions. Science. 2007;318:245-50.

37. Rensing SA, Lang D, Zimmer AD, Terry A, Salamov A, Shapiro H, et al. The Physcomitrella genome reveals evolutionary insights into the conquest of land by plants. Science. 2008;319:64-9.

38. Cannon SB, Mitra A, Baumgarten A, Young ND, May G. The roles of segmental and tandem gene duplication in the evolution of large gene families in Arabidopsis thaliana. BMC Plant Biol. 2004:4:1-21.
39. Li Z, Jiang $H$, Zhou $L$, Deng $L$, Lin $Y$, Peng $X$, et al. Molecular evolution of the HD-ZIP I gene family in legume genomes. Gene. 2014;533:218-28.

40. Verma JK, Gayali S, Dass S, Kumar A, Parveen S, Chakraborty S, et al. OsAlba1, a dehydration-responsive nuclear protein of rice (Oryza sativa L. ssp. indica), participates in stress adaptation. Phytochemistry. 2014;100:16-25.

41. Koutinas N, Pepelyankov G, Lichev V. Flower induction and flower bud development in apple and sweet cherry. Biotechnol Biotechnol Equip. 2010;24:1549-58

42. Giovannoni JJ. Genetic regulation of fruit development and ripening. Plant Cell. 2004;16:170-80.

43. Giovannoni JJ. Fruit ripening mutants yield insights into ripening control. Curr Opin Plant Biol. 2007;10:283-9.

44. Lemaire-Chamley M, Petit J, Garcia V, Just D, Baldet P, Germain V, et al. Changes in transcriptional profiles are associated with early fruit tissue specialization in tomato. Plant Physiol. 2005;139:750-69.

45. Banerjee A, Roychoudhury A. Abscisic-acid-dependent basic leucine zipper (bZIP) transcription factors in plant abiotic stress. Protoplasma. 2017:254:3-16.

46. Hernández Y, Sanan-Mishra N. miRNA mediated regulation of NAC transcription factors in plant development and environment stress response. Plant Gene. 2017;11:190-8.

47. Qin F, Shinozaki K, Yamaguchi-Shinozaki K. Achievements and challenges in understanding plant abiotic stress responses and tolerance. Plant Cell Physiol. 2011;52:1569-82

48. Saibo NJ, Lourenço T, Oliveira MM. Transcription factors and regulation of photosynthetic and related metabolism under environmental stresses. Ann Bot. 2009;103:609-23.

49. Goyal M, Alam A, Iqbal MS, Dey S, Bindu S, Pal C, et al. Identification and molecular characterization of an Alba-family protein from human malaria parasite plasmodium falciparum. Nucleic Acids Res. 2012:40:1174-90.

50. Huang YC, Niu CY, Yang CR, Jinn TL. The heat stress factor HSFA6b connects $A B A$ signaling and $A B A-$ mediated heat responses. Plant Physiol. 2016;172:1182-99.

51. Luo DL, Ba LJ, Shan W, Kuang JF, Lu WJ, Chen JY. Involvement of WRKY transcription factors in abscisic-acid-induced cold tolerance of banana fruit. J Agric Food Chem. 2017;65:3627-35.

52. Suzuki N, Bassil E, Hamilton JS, Inupakutika MA, Zandalinas SI, Tripathy $D$, et al. ABA is required for plant acclimation to a combination of salt and heat stress. PLoS One. 2016:11:e0147625.

53. Li C, Zhang B. MicroRNAs in control of plant development. J Cell Physiol. 2016;231:303-13.

54. Filipowicz W, Bhattacharyya SN, Sonenberg N. Mechanisms of posttranscriptional regulation by microRNAs: are the answers in sight? Nat Rev Genet. 2008:9:102-14.

55. Goodstein DM, Shu S, Howson R, Neupane R, Hayes RD, Fazo J, et al. Phytozome: a comparative platform for green plant genomics. Nucleic Acids Res. 2012;40:D1178-86

56. Mueller LA, Solow TH, Taylor N, Skwarecki B, Buels R, Binns J, et al. The SOL Genomics Network. A comparative resource for Solanaceae biology and beyond. Plant Physiol. 2005;138:1310-7.

57. Poole RL. The TAIR database. Methods Mol Biol. 2007;406:179-212.

58. Marchler-Bauer A, Derbyshire MK, Gonzales NR, Lu S, Chitsaz F, Geer LY, et al. CDD: NCBI's conserved domain database. Nucleic Acids Res. 2015:43:D222-6.

59. Schultz J, Milpetz F, Bork P, Ponting CP. SMART, a simple modular architecture research tool: identification of signaling domains. Proc Natl Acad Sci. 1998:95:5857-64

60. Guo AY, Zhu QH, Chen X, Luo JC. GSDS: a gene structure display server. Yi Chuan. 2007;29:1023-6.

61. Gasteiger E, Hoogland C, Gattiker A, Wilkins MR, Appel RD, Bairoch A. Protein identification and analysis tools on the ExPASy server. In: The proteomics protocols handbook; 2005. p. 571-607.

62. Sievers F, Wilm A, Dineen D, Gibson TJ, Karplus K, Li W, et al. Fast, scalable generation of high-quality protein multiple sequence alignments using Clustal Omega. Mol Syst Biol. 2011:7:539.

63. Robert $X$, Gouet P. Deciphering key features in protein structures with the new ENDscript server. Nucl Acids Res. 2014;42:320-4.

64. Bailey TL, Boden M, Buske FA, Frith M, Grant CE, Clementi L, et al. MEME SUITE: tools for motif discovery and searching. Nucl Acids Res. 2009:37:202-8. 
65. Horton P, Park KJ, Obayashi T, Fujita N, Harada H, Adams-Collier CJ, et al. WoLF PSORT: protein localization predictor. Nucl Acids Res. 2007;35:585-7.

66. Tamura K, Stecher G, Peterson D, Filipski A, Kumar S. MEGA6: molecular evolutionary genetics analysis version 6.0. Mol Biol Evol. 2013;30:2725-9.

67. Chen C, Chen H, Zhang Y, Thomas HR, Frank MH, He Y, et al. TBtools: an integrative toolkit developed for interactive analyses of big biological data. Mol Plant. 2020;13:1194-202.

68. Nei M, Gojobori T. Simple methods for estimating the numbers of synonymous and nonsynonymous nucleotide substitutions. Mol Biol Evol. 1986;3:418-26.

69. Nekrutenko A, Makova KD, Li WH. The KA/KS ratio test for assessing the protein-coding potential of genomic regions: an empirical and simulation study. Genome Res. 2002;12:198-202.

70. Koch MA, Haubold B, Mitchell-Olds T. Comparative evolutionary analysis of chalcone synthase and alcohol dehydrogenase loci in Arabidopsis, Arabis, and related genera (Brassicaceae). Mol Biol Evol. 2000;17:1483-98.

71. Yang J, Yan R, Roy A, Xu D, Poisson J, Zhang Y. The I-TASSER suite: protein structure and function prediction. Nat Methods. 2015;12:7-8.

72. Xu D, Zhang Y. Improving the physical realism and structural accuracy of protein models by a two-step atomic-level energy minimization. Biophys J. 2011;101:2525-34.

73. Kim SR, Lee DY, Yang Jl, Moon S, An G. Cloning vectors for rice. J Plant Biol. 2009;52:73.

74. Wai AH, Waseem M, Khan AB, Nath UK, Lee DJ, Kim ST, et al. Genomewide identification and expression profiling of the PDI gene family reveals their probable involvement in abiotic stress tolerance in tomato (Solanum lycopersicum L.). Genes. 2021;12:23.
75. Balestrini R, Gómez-Ariza J, Lanfranco L, Bonfante P. Laser microdissection reveals that transcripts for five plant and one fungal phosphate transporter genes are contemporaneously present in arbusculated cells. Mol Plant-Microbe Interact. 2007;20:1055-62.

76. Schmittgen TD, Livak KJ. Analyzing real-time PCR data by the comparative CT method. Nat Protoc. 2008;3:1101-8.

77. Andrews S. FastQC: a quality control tool for high throughput sequence data. Cambridge: Babraham Bioinformatics, Babraham Institute; 2010.

78. Chen S, Zhou Y, Chen Y, Gu J. fastp: an ultra-fast all-in-one FASTQ preprocessor. Bioinformatics. 2018;34:1884-90.

79. Kim D, Paggi JM, Park C, Bennett C, Salzberg SL. Graph-based genome alignment and genotyping with HISAT2 and HISAT-genotype. Nat Biotechnol. 2019;37:907-15.

80. Liao Y, Smyth GK, Shi W. featureCounts: an efficient general-purpose read summarization program. Bioinformatics. 2014;30:923-30.

81. Smyth GK. Limma: linear models for microarray data. In: Bioinformatics and computational biology solutions using R and Bioconductor. New York: Springer; 2005. p. 397-420

82. Langfelder P, Horvath S. WGCNA: an R package for weighted correlation network analysis. BMC Bioinformatics. 2008;9:1-3.

83. Törönen P, Medlar A, Holm L. PANNZER2: a rapid functional annotation web server. Nucleic Acids Res. 2018:46:W84-8.

84. Moriya Y, Itoh M, Okuda S, Yoshizawa AC, Kanehisa M. KAAS: an automatic genome annotation and pathway reconstruction server. Nucleic Acids Res. 2007;35:W182-5.

\section{Publisher's Note}

Springer Nature remains neutral with regard to jurisdictional claims in published maps and institutional affiliations.
Ready to submit your research? Choose BMC and benefit from:

- fast, convenient online submission

- thorough peer review by experienced researchers in your field

- rapid publication on acceptance

- support for research data, including large and complex data types

- gold Open Access which fosters wider collaboration and increased citations

- maximum visibility for your research: over $100 \mathrm{M}$ website views per year

At BMC, research is always in progress.

Learn more biomedcentral.com/submissions 